\title{
Extravehicular Activity Operations Concepts under Communication Latency and Bandwidth Constraints
}

\author{
Kara H. Beaton \\ KBRwyle Science, Technology and \\ Engineering Group \\ KBRwyle/HAC/37C \\ 2400 NASA Parkway \\ Houston, TX 77058 \\ kara.h.beaton@nasa.gov \\ Matthew J. Miller \\ Georgia Institute of Technology \\ 270 Ferst Drive, Room 416 \\ Atlanta, GA 30332 \\ mmiller@gatech.edu
}

\author{
Steven P. Chappell \\ KBRwyle Science, Technology and \\ Engineering Group \\ KBRwyle/HAC/37C \\ 2400 NASA Parkway \\ Houston, TX 77058 \\ steven.p.chappell@nasa.gov \\ Shannon Kobs Nawotniak \\ Idaho State University \\ Department of Geosciences \\ 921 S. $8^{\text {th }}$ Ave, Mail Stop 8072 \\ Pocatello, ID 83209 \\ kobsshan@isu.edu
}

\author{
Andrew F. J. Abercromby \\ National Aeronautics and \\ Space Administration \\ 2101 NASA Parkway \\ Houston, TX 77058 \\ andrew.abercromby@nasa.gov
}

\author{
Allyson Brady \\ McMaster University \\ School of Geography \& Earth Sciences \\ General Science Building Room 315 \\ Hamilton, Ontario L8S4L8 Canada \\ bradyal@mcmaster.ca
}

\author{
Darlene S. S. Lim \\ NASA Ames Research Center \\ Mail-Stop 245-3 \\ Moffett Field, CA 94035 \\ darlene.lim@nasa.gov
}


flows are being investigated through multiple field deployments at two high-fidelity Mars analog locations: the Southwest and East Rift Zone flows on the Big Island of Hawai' $i$ (reminiscent of "early Mars," when basaltic volcanism and interaction with water were widespread), and the Eastern Snake River Plane Craters of the Moon (COTM) National Monument in Idaho (as an analog to "present-day Mars," where basaltic volcanism is rare and most evidence for volcano-driven hydrothermal activity is relict) (Figure 1 and Figure 2). The BASALT Science primary research question is: How do microbial communities and habitability correlate with the physical and geochemical characteristics of chemically altered basalt environments? Specifically, we are interested in:

Science 1A. What are the geochemical, mineralogical, and textural properties associated with basalts affected by liquid water, intrinsic volatiles, and fumarolic gases at complementary Mars analog sites?

Science 1B. What geochemical and geological conditions provide appropriate energy sources, major biogenic elements (CHNOPS), liquid water, and micro-habitats for microbial growth?

Science 2A. What is the relationship between the physical characteristics and geochemistry of Mars analog basalts and the biomass that they can support?

Science 2B. What are the upper bounds on the biomass that could have been supported on Mars?

Science 2C. How does this upper bound inform future requirements to detect extinct life on Mars?

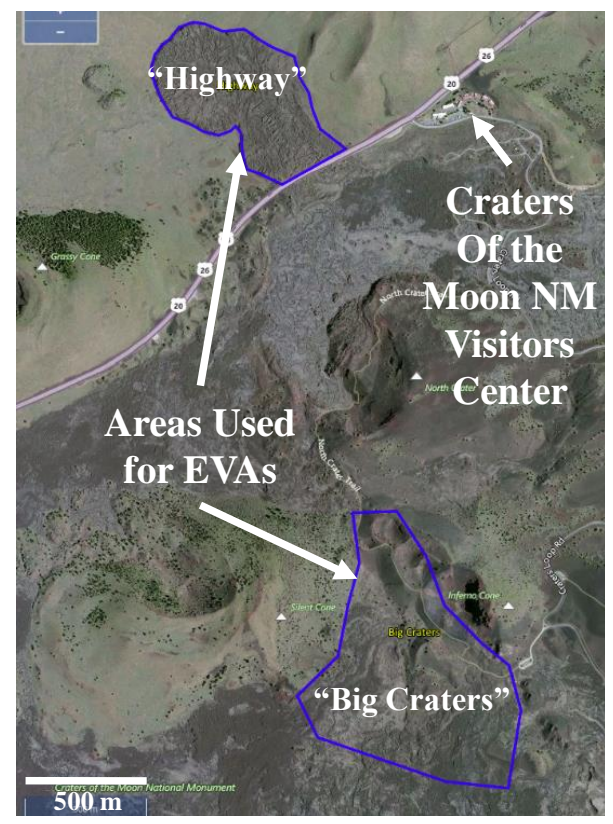

Figure 1. BASALT-1 analog environment for presentday Mars: COTM Highway and Big Craters Flows

Scientific fieldwork is being conducted under simulated Mars mission constraints based on current architectural assumptions for future Mars exploration missions [1]. Specifically, the BASALT project is evaluating communication latencies of 5 and 15 min one-way light time (OWLT), which fall within the 4-22 min OWLT delays experienced between
Mars and Earth, a low-bandwidth condition of $0.512 \mathrm{Mb} / \mathrm{s}$ uplink and $1.54 \mathrm{Mb} / \mathrm{s}$ downlink, representing a conservative and affordable flight data rate, and a high-bandwidth condition of $5.0 \mathrm{Mb} / \mathrm{s}$ uplink and $10.0 \mathrm{Mb} / \mathrm{s}$ downlink, representing an upgraded human mission capability that would require additional infrastructure and technology development. The BASALT Science Operations primary research question is: Which exploration ConOps and capabilities enable and enhance scientific return during human-robotic exploration under Mars mission constraints? More specifically:

Science Ops 1A. Do the baselined Mars mission ConOps, software systems, and communication protocols developed and tested during previous NASA analog tests work acceptably during real scientific field exploration? What improvements are desired, warranted, or required?

Science Ops 1B. Do these ConOps, software systems, and communication protocols remain acceptable as communication latency increases from 5 to $15 \mathrm{~min}$ OWLT? What improvements are desired, warranted, or required?

Science Ops 2A. Which capabilities are enabling and significantly enhancing for Mars scientific exploration?

Science Ops 2B. Do these capabilities remain enabling and significantly enhancing as communication latency increases from 5 to 15 min OWLT?

Science Ops 2C. Do these capabilities remain enabling and significantly enhancing as communication bandwidth allowances decrease?

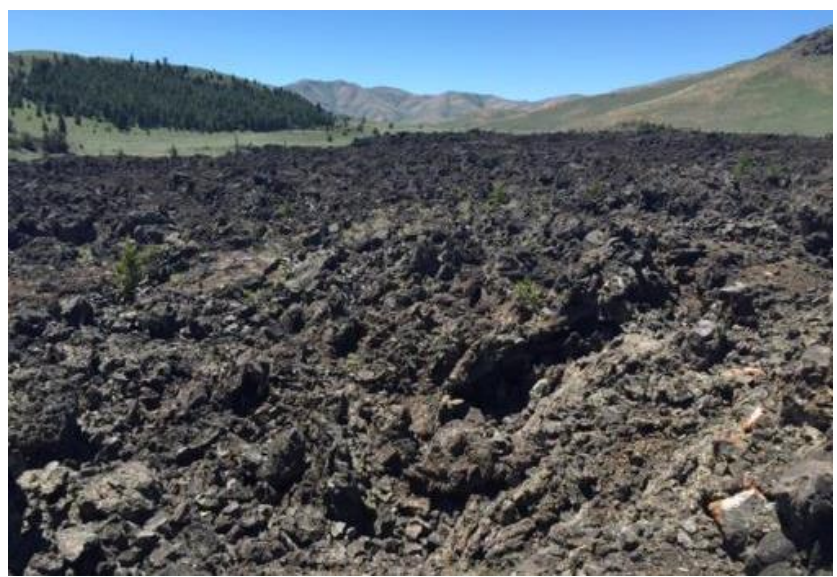

Figure 2. Representative lava field in COTM explored during BASALT-1

The BASALT project also incorporates relevant technologies and science support tools to aid in effective and efficient mission planning, scheduling, navigation, task execution and documentation, decision making, and communication between "Mars" and "Earth." Many of these capabilities are accomplished through a suite of complementary science operations tools that are collectively referred to as Minerva. Minerva includes the Exploration Ground Data System (xGDS), a software package that enables science operations planning, monitoring, documenting, archiving, and searching [2], Playbook, an advanced timeline tracking tool with text messaging 
capabilities [3], and SEXTANT, a traverse optimization planning tool [4]. Additional technologies for field operations include custom designed EVA informatics backpacks, which provide voice, video, and GPS positions from the extravehicular (EV) crewmembers, and EVA graphical wrist displays, so that the EV crew can view their traverses, video camera data, and important text messages from Earth.

This paper focuses on the Science Operations architecture for the BASALT project, as well as the Science Operations research questions $1 \mathrm{~A}$ and $1 \mathrm{~B}$ results and lessons learned from the first field deployment, referred to as BASALT-1, which took place at COTM in June of 2016.

\section{EVA Personnel and Communication Infrastructure}

The BASALT baseline ConOp stems from the results of previous analog studies, including the Desert Research And Technology Studies (DRATS) [5-7], NASA Extreme Environment Mission Operations (NEEMO) [8-10], and the Pavilion Lake Research Project (PLRP) $[11,12]$. Our baseline architecture includes 2 "Mars" EV crewmembers in the field completing the science tasks, 2 "Mars" intravehicular (IV) crewmembers supporting the EV crew and communicating with "Earth" from an IV workstation (inside a simulated rover or habitat [7, 13]), and an "Earth" Mission Support Center (MSC) that provides scientific expertise and operational guidance across communication latency and bandwidth limitations [1, 10, 14]. Table 1 describes the key personnel and their respective roles and responsibilities.

Our communication infrastructure supports the transmission of continuous voice, continuous video, continuous GPS position tracks of the EV crew, still imagery, and text data between the EV and IV crewmembers at near-zero latency under high bandwidth conditions, simulating communication among crew co-located on Mars. These data products are also transmitted directly to the MSC across latency under either high or low bandwidth conditions (depending on the particular bandwidth condition being evaluated during that EVA). The main two-way communication path between the MSC and the EV crew is through the IV crewmembers; namely, the CAPCOM (capsule communicator) and SCICOM (science communicator) converse directly with IV1 and IV2 (across time delay), who then relay the relevant information (at the appropriate time) to EV1 and EV2.

Two primary voice communication loops are employed during BASALT EVAs: space-to-ground-1 (S2G1), across which the EV and IV crew talk with one another in real-time, and space-to-ground-2 (S2G2), in which the IV crew and the MSC communicate across time delay. The S2G1 loop is transmitted to the MSC across delay so that the MSC can hear the EV-IV crew conversations. EV crewmembers do not listen to the S2G2 loop. In general, the S2G2 loop is used infrequently, especially in the MSC-to-IV direction, as it can be challenging to receive voice messages "from the past;" however, there are instances when this loop is helpful, such as when used as added redundancy to ensure an important text message is not missed.
Text messaging during the EVAs is provided by the Playbook Mission Log [3]. The Mission Log supports texting in realtime between the EV and IV crewmembers (although this capability is rarely utilized due to the EV crew needing their hands free for science tasks in the field) and also across time delay between the IV crew and the MSC (as the primary means of communication between Mars and Earth). EV crewmembers interact with the Mission Log on their graphical wrist displays, but usually only do so if prompted by the IV crewmembers (such as when an annotated image from the MSC is posted that points to a specific feature of interest).

Still images, video streams, and GPS position tracks from the field are relayed to the IV crewmembers in real-time and to the MSC (as bandwidth allows) across time delay through $x$ GDS. This data is automatically archived in the xGDS database and linked to the current EVA. Relevant tags and descriptive notes can be appended to each still image by the IV crew and by members of the MSC.

\section{Table 1. BASALT key roles and responsibilities}

Mars 2 EV crewmembers: in the field cooperatively Crew completing science tasks; EV1 (operations EV crewmember) leads timeline management, traverse navigation, and other operational tasks, while EV2 (EVA science lead) leads the science execution.

2 IV crewmembers: inside an IV workstation guiding the EVAs; IV1 (EVA operations lead) primarily interacts with the EV crew and MSC (via CAPCOM) on operational tasks, timelines, constraints, and procedures, while IV2 (science IV crewmember) primarily interacts with the EV crew and MSC (via SCICOM) on science tasks, priorities, and recommendations.

Earth Fight Director: has authority over all operational

MSC recommendations from the MSC.

Science Team Lead: has authority over all scientific recommendations from the MSC; leads science team in providing tactical feedback to EV/IV crew.

CAPCOM: communicates with IV1 on operational tasks, timeline, constraints, and procedures.

SCICOM: communicates with IV2 on science tasks, priorities, and recommendations; tracks EVA timeline and keeps Science Team apprised of critical bingo times based on current communication latency.

EVA Planner: monitors and updates timeline based on EV crew progress; assists SCICOM with tracking critical bingo times.

Science Team Members: science experts that tactically and strategically plan and guide EVA execution.

\section{EVA Traverse and Timeline Design}

For destinations such as Mars, it is assumed that robotic precursor missions will have collected sufficient high-quality 
imagery and precursor data to plan EVA traverses to be explored by human crews [7]. BASALT-1 precursor data included Google Earth imagery at a resolution of $10 \mathrm{~m}$ per pixel. BASALT scientists used this information to identify candidate locations of scientific interest, referred to as EVA stations. Each station was approximately $10 \mathrm{~m}$ diameter in size. Stations were then grouped and organized into baseline (planned) EVA traverses (the routes between and within stations).

It is also assumed the EV crew "boots-on-the-ground" perspective will provide more scientific information than can be obtained from precursor data [15]. This includes, for example, the ability to collect higher resolution imagery from additional angles and the ability to extract surface samples for more detailed investigations; this additional data can also lead to modified traverse plans, science tasks, and science priorities. Additionally, however, it is assumed that more scientific expertise will consistently reside on Earth than with the crew [16]. While Mars crewmembers will be extensively trained, the multitude of science objectives that will fill the long-duration Mars surface missions may be more successfully met if strategic Mars-to-Earth interactions can be accommodated [16]. Even Apollo astronauts, who had significant training in geology and science tasks before their relatively short-duration missions [17], were supported by an Earth-based team of expert scientists who were essential to the overall scientific success of the missions [18-20]. Both of these assumptions can impact inter- and intra-EVA timeline design.

During Apollo, the OWLT communication latency between the Earth and the Moon was minimal ( 1.25 s), which allowed for meaningful, near real-time interaction between the astronauts and scientists during the EVAs without special consideration for data transmission times; hence there were minimal losses in efficiency or increases in crew idle time (defined as the time spent waiting for input from Earth) [21]. However, as communication latency increases for destinations such as Mars and bandwidth limitations restrict the amount of data (including voice, video, still imagery, text messages, and scientific instrument data) that can be transmitted between Mars and Earth, achieving meaningful input from Earth during EVAs will be more difficult [22]. Based on these challenges, one Mars exploration ConOp could implement a nearly autonomous crew to execute the science objectives with an Earth-based MSC acting primarily as a passive observer who only provides opportunistic feedback across latency and under bandwidth constraints during the EVA as able. In this case, the MSC would mainly provide strategic input between EVAs, as opposed to within EVAs. An alternate ConOp could implement strategically designed EVA timelines with built-in timing accommodations to allow for the crew to transmit science data to the MSC so that they can analyze and interpret this data prior to sending guidance back to the crew for subsequent EVA tasks. This alternative ConOp does not preclude the first ConOp, but also adds the opportunity for tactical MSC input to actively influence intra-
EVA execution. While both ConOps offer scientific (and operational) advantages, the BASALT project focuses on the later.

Enabling intra-EVA interactions between Mars and Earth under communication latency and bandwidth limitations requires special consideration be given to the design of the EVA timeline (the sequence of tasks to be performed along the traverse). To minimize crew idle time, there must be a clear delineation between EVA tasks that can be done independent of Earth input and tasks that are either dependent on or could substantially benefit from Earth input. For tasks benefiting from Earth input, dependent task groups can be created and distributed throughout the timeline; other tasks in the timeline can be decoupled from the dependent task group(s) and may be performed stand-alone. For instance, a dependent task pair could consist of a pre-sampling survey (e.g., contextual descriptions, still imagery, and video footage) and a corresponding sampling task at a particular location of interest. The EV crew could complete the pre-sampling survey and send that data to the MSC. The MSC could use this information to guide the sampling (including details regarding where, how much, etc.). While the MSC is formulating their sampling plan based on this pre-sampling information and information is flowing between Mars and Earth across latency, the EV crew can complete second pre-sampling survey or a separate stand-alone task. With sufficient understanding of EVA task dependencies, task durations, communication latencies, and ground assimilation time (GAT, the amount of time needed by the ground to provide meaningful input for dependent tasks), timelines can be created that allow for Earth input on many or most tasks while minimizing or avoiding crew idle time [10]. BASALT timelines were strategically designed to enable interactions between crewmembers and the MSC and to minimize crew idle time.

\section{METHODS}

\section{Science Operations Study Design}

The BASALT-1 field deployment consisted of a series of 10 simulated EVAs in which scientifically significant samples of basalt were extracted from "Mars" (i.e., COTM) by the EV crewmembers, who were guided by the IV crew and the MSC during the EVAs. Each EVA was conducted under one of the 4 communication study conditions: 5 or $15 \mathrm{~min}$ latency, high or low bandwidth. The BASALT-1 planned schedule assigned 2 EVAs to each study condition, leaving the last two EVAs initially unassigned to account for potential contingencies that arose during the field deployment. Two EVA teams (A and B) were established based on the key roles described in Table 1, including 2 pairs of EV and IV crewmembers, 2 Flight Directors, 2 Science Team leads, and (due to limited operations personnel) 1 SCICOM and 1 CAPCOM who were part of both teams. Each team was scheduled to experience each study condition at least once. See Error! Reference 
source not found. in Section 3 for the planned EVA schedule.

Data passed between EV and IV crewmembers and between Mars and Earth included EV and IV voice communication channels, video footage from the EV crew video cameras, video footage from a mobile situational awareness (SA) camera, still imagery captured by the EV crew, GPS position tracks of the EV crew, physiological monitoring data of the EV crew, text messages between the IV crew and the MSC, scientific field notes, and annotated images between the MSC and the crew. For this field deployment, scientific instrument data was communicated through still images of the relevant instrument results screens. The data rates and resolutions associated with each of these capabilities were selected such that all capabilities were utilized in full during EVAs conducted under the high bandwidth study condition. EVAs executed under the low bandwidth condition did not pass any video data from the $\mathrm{EV}$ crew to the MSC, and the rate and resolution of still images and text messages between the crew and the MSC were limited to $0.512 \mathrm{Mb} / \mathrm{s}$ uplink and 1.54 $\mathrm{Mb} / \mathrm{s}$ downlink. Throughout each EVA, network analytics were run in real-time to ensure bandwidth traffic stayed within the high and low bandwidth constraints defined above.

\section{EVA Field Equipment and Facilities}

During the EVAs, the EV crewmembers wore custom informatics backpacks (Figure 3) designed by the BASALT Backpacks Team, which housed the hardware that enabled twoway voice communication with the IV crew and one-way transmission of video, still imagery, GPS position data, and physiological monitoring data to the IV crew and the MSC. Each EV crewmember wore a Zephyr BioHarness that tracked heart rate, respiration, and kinematic movements, and also a wrist-mounted display (Apple iPhone 6) that showed the planned and actual traverses, pins marking significant locations along the traverse, the view finder for their video cameras, and the Mission Log. A mobile SA camera, simulating a mast-mounted rover camera, was set up at each station by the EV crew and provided situational awareness of their location within the surrounding terrain. Handheld scientific field instruments included a Fourier transform infrared (FTIR) spectroscopy instrument and a near infrared (NIR) spectrometer. Sampling tools included sterile gloves, rock sledges, chisels, and sample bags.

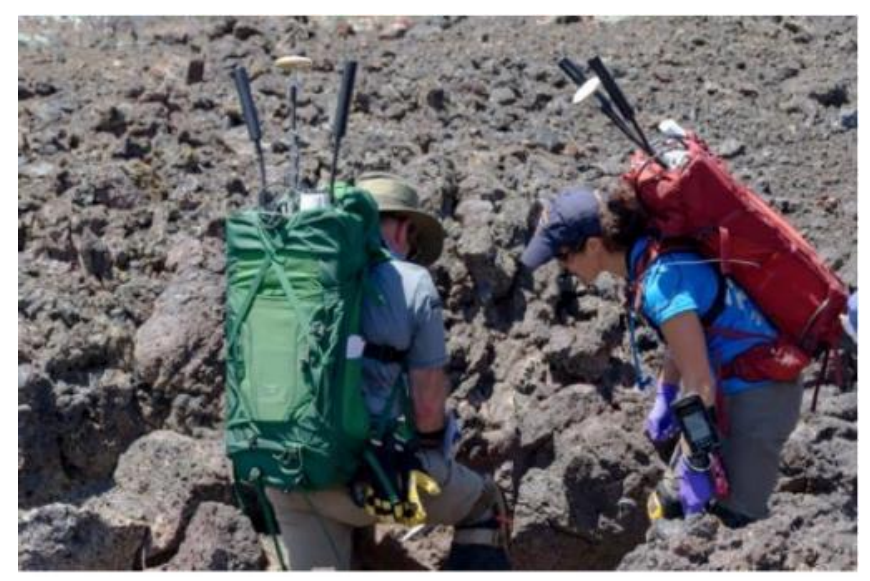

Figure 3. BASALT-1 EV crewmembers in the field wearing the informatics backpacks and wrist displays

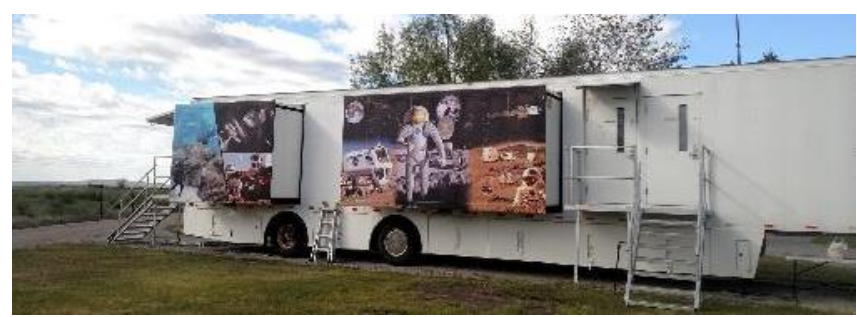

Figure 4. BASALT-1 trailer that housed the IV workstation and MSC workspace during the EVAs

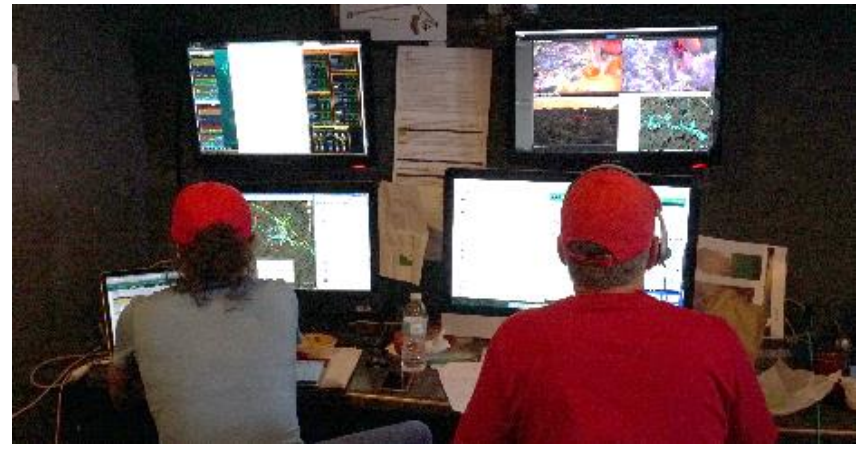

Figure 5. BASALT-1 IV workstation in use during an EVA by IV1 (left) and IV2 (right)

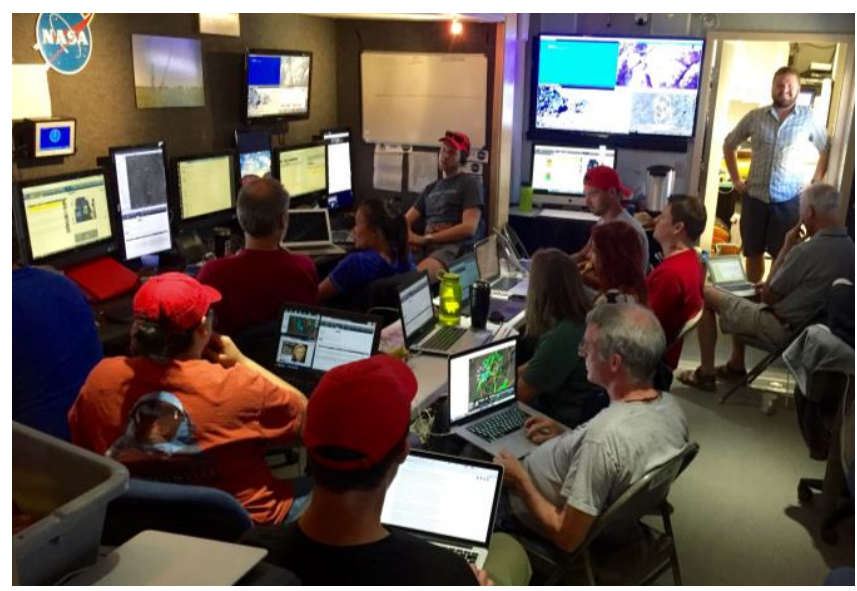

Figure 6. BASALT-1 MSC workspace 
The BASALT Communications Team provided a trailer to house the IV crewmembers and the MSC (Figure 4). The IV crew and MSC were physically separated by a closed door during the EVAs. An IV workstation was established inside the trailer, which included a laptop computer and 2 additional display screens for each IV crewmember (Figure 5). Audio headsets with push-to-talk capabilities were used for voice communication with the EV crewmembers (across the S2G1 communication loop) and with the MSC (across S2G2). The MSC consisted of 2 rows of tables to accommodate individual laptops for all MSC members, additional display screens for the Flight Director, Science Team Lead, SCICOM, and several science team members, and a central computer with large external display for all members of the MSC to view (Figure 6). The BASALT Communication and Backpack teams established network connectivity from the EV informatics backpacks and mobile SA camera to the IV workstation and MSC through the use of fixed antennae and mobile repeaters located between the field sites and the BASALT trailer.

\section{EVA Planning and Execution}

Prior to the BASALT-1 field deployment, several multi-day engineering readiness tests (ERTs) were completed to test the individual components of the BASALT hardware and software. ERTs took place at NASA Kennedy Space Center, where the BASALT Communications and Backpack Team are located, and at NASA Ames Research Center (ARC), where the Minerva team is located. Following the ERTs, multiday operational readiness tests (ORTs) were conducted at NASA ARC to integrate all hardware, software, and communications infrastructure, train personnel in the key roles outlined in Table 1, and to practice scientific field operations. All EV crewmembers arrived several days early to the BASALT-1 field deployment for additional training in the actual basalt terrain.

Table 2 .
The MSC used xGDS and SEXTANT to plan the BASALT1 EVA traverses. Each EVA included 2 stations of scientific interest, and the traverse included the route from a pre-defined starting location, through each of the stations, and then back to the start. EV crewmembers studied these traverses prior to each EVA. Figure 7 shows a representative planned traverse from one of the BASALT-1 EVAs.

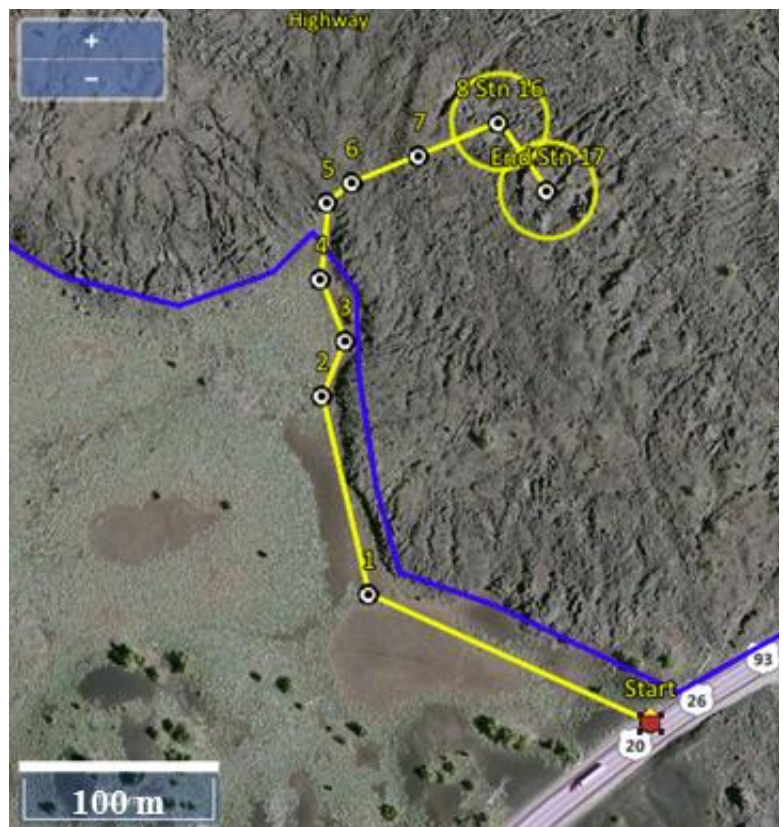

Figure 7. Representative planned BASALT-1 EVA traverse; small white circles are navigation waypoints and larger yellow circles are stations

Flight rules were established to govern all aspects of the BASALT-1 field operations and provided the operating guidelines with respect to safety, mission management and authority, EVA management and authority, troubleshooting, and ground rules. The flight rules are listed in

EVA timelines incorporated five phases: approach, contextual survey, sample location search, pre-sampling survey, and sampling. A representative planned EVA timeline is outlined in

Throughout the EVA, the MSC monitored and reviewed incoming data from the field across delay, recorded additional field notes, and provided recommendations for pre-sampling and sampling based on their collective expertise (Figure 9). The science team utilized dynamic priority ranking lists, referred to as dynamic leaderboards, to track and rank candidate samples relative to one another and against the science objectives for the current EVA [12]. The science team build the dynamic leaderboards by integrating and interpreting the incoming verbal descriptions, still imagery, video footage, and instrument data from the field. Updates to the dynamic leaderboards were relayed regularly to the IV crew via the Mission Log, who could then discuss these rankings with the EV crew. The use of these leaderboards enabled the crew to track the dynamic nature of MSC recommendations and helped minimize crew idle time since as soon as a new candidate sample marker was laid down by the EV crew, the MSC could update the leaderboard and relay that information to the crew (across latency). Dynamic leaderboards were built for both the pre-sampling and sampling phases of the EVA. 


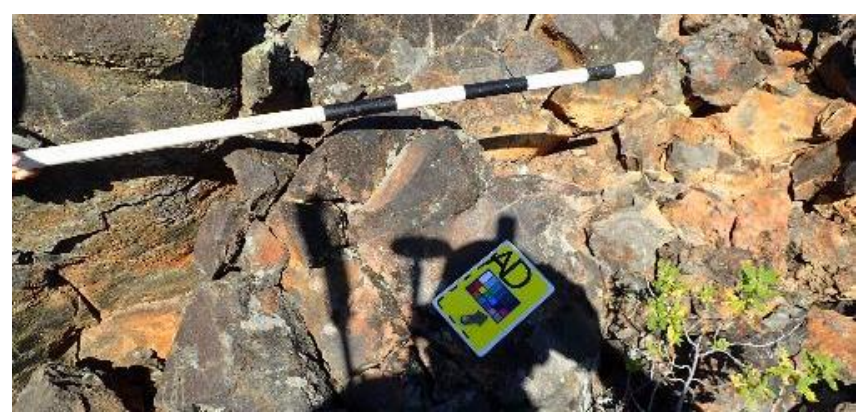

Figure 8. Temporary tags used to mark candidate sample locations; tags included an alphanumeric label, color bar, scale bar, and rotatable north arrow

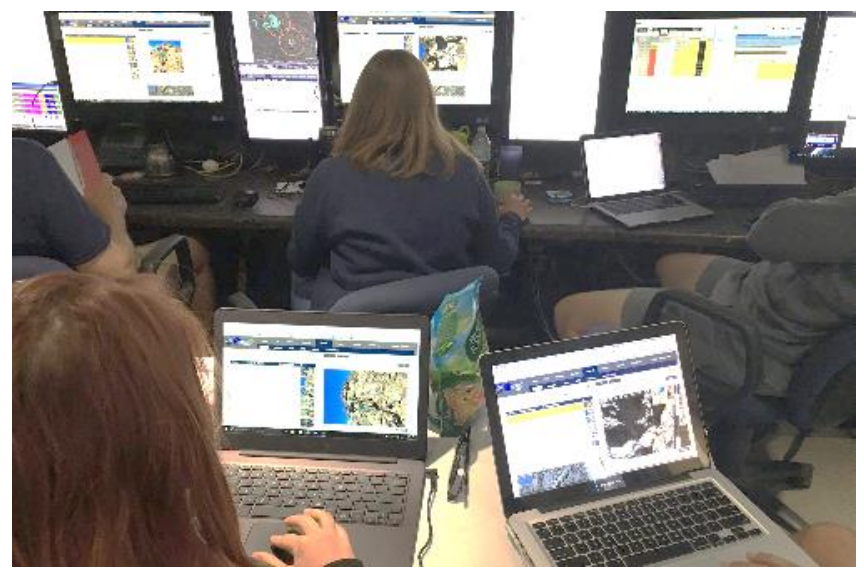

Figure 9. MSC members utilizing xGDS to record field notes and monitor video and still imagery during an EVA

In addition to the personnel roles detailed in Table 1, the MSC also included a Biology Lead, who provided feedback regarding features that may have an impact on habitability and/or the microbial community, a Geology Lead, who provided feedback on significant geological features, an Instrument Lead, who examined the spectral instrument data and offered additional information based on the instrument scans, an Imagery Lead, who carefully examined the details of the incoming still imagery, a Leaderboard Lead, who recorded the dynamic leaderboard priorities, alternatives, and rationale based on science team discussions, a Tactical Awareness Management Lead, who kept track of exactly where the EV crew were in the EVA timeline, and a Strategic Awareness Management Lead, who maintained general situational awareness in the context of the overall mission objectives and how the ongoing EVA activities may influence future EVAs.

\section{Out-of-Simulation EVA Support Personnel}

Each EVA was supported by a network of out-of-simulation support personnel. A Field Support Team (FST) assisted the EV crewmembers in the field and carried the science instruments and sampling tools. The FST Lead was responsible for leading the FST and coordinating all out-of-simulation activities, including those that occurred before, during, and after the EVA. The FST Instrument Aid provided the EV crew with technical instrument support. The FST Biology Sterilization Aid assisted the EV crew with maintaining sterilization during sampling tasks. The FST Steno/Runner recorded detailed field notes in the event of communication dropouts and assisted the FST Lead as needed. Two members of the BASALT Communications and Backpack Team provided communication infrastructure and backpack troubleshooting support in the field.

Inside the MSC, the simulation coordinator (SIMCOORD) communicated with the FST Lead to coordinate the start and end of each EVA. The MSC was further staffed with additional communications infrastructure and Minerva technical support personnel.

Table 3. EV crewmembers began each EVA with a local report, which included the local time, wind speed and direction, percentage cloud cover, sun angle, temperature, and precipitation. During their first station approach, the EV crew traversed along the recommended route while providing verbal descriptions of the surrounding topography and capturing corresponding still imagery and video footage, as allowed by bandwidth conditions. Along the way, the crew also looked for targets of opportunity (TOPs): locations of potentially significant scientific interest that were not part of the planned EVA traverse, but may be worth further investigation later in the current EVA or during a subsequent EVA. Upon arriving at the perimeter of the first station, EV crew completed a station contextual survey, which consisted of positioning the mobile SA camera and providing a contextual report that included discussion on the general orientation, shape, condition, and color of the station's basalt, the presence of water, fluids, and biomass, and any other relevant details that might be significant for meeting the science objectives for that EVA. The EV crew then proceeded into a sample location search in which they searched for candidate samples of basalt that met the current science priorities. Temporary tags were used to mark candidate sample locations, which were approximately $1 \mathrm{~m}$ in size, and serve as communication references (Figure 8). EV crewmembers then provided verbal descriptions of each candidate and collected corresponding still imagery, video footage (as bandwidth allowed), and forward looking infrared (FLIR) thermal data. Following the completion of the sample location search at the first station, the EV crew then traversed to the second station, where they completed another approach, contextual survey, and sample location search. The crew then proceeded into the pre-sampling survey, in which they returned to the candidate sample locations recommended by the MSC as the highest potential sampling priority locations to identify candidate replicates (specific portions of the basalt within the sample location that could be extracted during the sampling phase), collect additional detailed still imagery and video footage (as bandwidth allowed), and obtain Fourier transform infrared (FTIR) spectroscopy and near infrared (NIR) spectrometer instrument scans (for mineral identification) of representative replicates. After completing the pre-sampling survey, the EV crew collected samples based on guidance from the MSC. A full sampling suite was typically collected, which included 7 samples ranging in size from approximately $5-15 \mathrm{~cm}$ in diameter that would be further analyzed by BASALT scientists post-deployment. 
During each EVA phase, the IV crewmembers assisted the EV crew through the timeline tasks in real-time and conversed (primarily via text messages and recorded field notes) with the MSC across delay. IV1 focused on the operational aspects of the EVA while IV2 focused on the detailed science. Specifically, IV1 ran a tactical EVA management tool (a timeline spreadsheet that enabled the crew to monitor planned verses actual task start times, end times, and durations and to project future task start times based on how far ahead or behind the EV crew were from the planned timeline) and reported relevant timing information to the EV crew, tracked GPS positions of the EV crew relative to the planned traverses and provided heading and distance information to the EV crew upon request, posted operationally relevant information to the Mission Log, verified incoming still imagery and added tags and notes to each image within xGDS, monitored EVA physiological data (including heart rate, respiration rate, and kinematic data), and monitored and responded to simulated EVA telemetry (including spacesuit consumable) data. IV2 formulated the master list of pre-sampling and sampling priorities based on recommendations from the EV crew and communication (primarily via the Mission Log) with the MSC; IV2 was also responsible for recording detailed scientific field notes in xGDS. Both IV1 and IV2 monitored the mobile SA and EV crewmember video feeds streaming from the field.

Table 2. BASALT-1 Flight Rules

\begin{tabular}{|c|c|}
\hline \multicolumn{2}{|l|}{ Safety } \\
\hline S1 & $\begin{array}{l}\text { Any person may stop an activity (in-sim or out-of-sim) at any time for any reason to ensure safety of personnel and } \\
\text { protection of the environment. }\end{array}$ \\
\hline \multicolumn{2}{|c|}{ Mission Management and Authority } \\
\hline MM1 & $\begin{array}{l}\text { The Mission Management Team (MMT) has authority and responsibility for strategic (e.g. EVA planning) deci- } \\
\text { sions affecting scientific and/or science operations objectives. Strategic decisions affecting science and/or science } \\
\text { operations objectives must be discussed with the MMT. }\end{array}$ \\
\hline MM2 & All EVA plans must be approved and finalized by the MMT at least 12 hours prior to execution. \\
\hline MM3 & $\begin{array}{l}\text { Minutes shall be taken by the Documentarian during all MMT meetings including documentation of all decisions } \\
\text { and plan changes. }\end{array}$ \\
\hline \multicolumn{2}{|c|}{ EVA Management and Authority } \\
\hline EM1 & $\begin{array}{l}\text { The crew has authority and responsibility for tactical (i.e. EVA execution) decisions: } \\
\text { - IV1: Authority and responsibility for operational EVA decisions and tactics. } \\
\text { - EV2: authority and responsibility for scientific EVA decisions and tactics. } \\
\text { - MSC (FD, CC, SBT are advisory only). }\end{array}$ \\
\hline EM2 & Flight Director has authority over all operational recommendations from the MSC. \\
\hline EM3 & Science Team Lead has authority over all scientific recommendations from the MSC. \\
\hline EM4 & CAPCOM / SCICOM is responsible for clear communication between the MSC and the crew. \\
\hline EM5 & "In-Sim" activities take priority over "out-of-sim" activities. \\
\hline EM8 & EVA durations shall not exceed 5 hours. \\
\hline EM9 & EVAs shall be planned to fit a $<=4$ hour timeline plus 30 mins margin. \\
\hline EM10 & $\begin{array}{l}\text { Extensions up to } 30 \text { additional min (beyond the } 30 \text { min margin called out in EM9 up to } 5 \text { hours total PET) can be } \\
\text { proposed no later than the planned end time of the EVA. Consent by all members is not required as long as the } \\
\text { FST, Communications Team, and EV/IV agree to the extension and the extension is formally stated to MSC. }\end{array}$ \\
\hline \multicolumn{2}{|c|}{ Troubleshooting } \\
\hline $\mathrm{T} 1$ & $\begin{array}{l}\text { Minimum Acceptable Communication Conditions: Once started, Simulations may continue under degraded com- } \\
\text { munication conditions until indicted otherwise by the EV crew (e.g. EV crew determine they cannot execute EVA } \\
\text { timeline without input from IV/MSC). }\end{array}$ \\
\hline $\mathrm{T} 2$ & $\begin{array}{l}\text { The Documentarian shall document the dates and times during which in-sim EVAs are conducted without specific } \\
\text { systems available. }\end{array}$ \\
\hline T3 & $\begin{array}{l}\text { Real-time position tracking and physiological monitoring are not required. EVA and EVA start times will not be } \\
\text { delayed or interrupted to permit troubleshooting of physiological sensors. }\end{array}$ \\
\hline T4 & $\begin{array}{l}\text { An established simulation not to exceed end time will be defined for each EVA (e.g. not to exceed simulation time } \\
\text { beyond 5:00 PM) by the MMT the day prior to operations. If troubleshooting may prohibit continuing of simula- } \\
\text { tions for that day, an impromptu MMT meeting will take place to establish priorities for the remainder of the day. }\end{array}$ \\
\hline \multicolumn{2}{|c|}{ Ground Rules } \\
\hline GR1 & Within the perimeter of the EVA subjects and EV support $d$ \\
\hline
\end{tabular}


Throughout the EVA, the MSC monitored and reviewed incoming data from the field across delay, recorded additional field notes, and provided recommendations for pre-sampling and sampling based on their collective expertise (Figure 9). The science team utilized dynamic priority ranking lists, referred to as dynamic leaderboards, to track and rank candidate samples relative to one another and against the science objectives for the current EVA [12]. The science team build the dynamic leaderboards by integrating and interpreting the incoming verbal descriptions, still imagery, video footage, and instrument data from the field. Updates to the dynamic leaderboards were relayed regularly to the IV crew via the Mission Log, who could then discuss these rankings with the EV crew. The use of these leaderboards enabled the crew to track the dynamic nature of MSC recommendations and helped minimize crew idle time since as soon as a new candidate sample marker was laid down by the EV crew, the MSC could update the leaderboard and relay that information to the crew (across latency). Dynamic leaderboards were built for both the pre-sampling and sampling phases of the EVA.

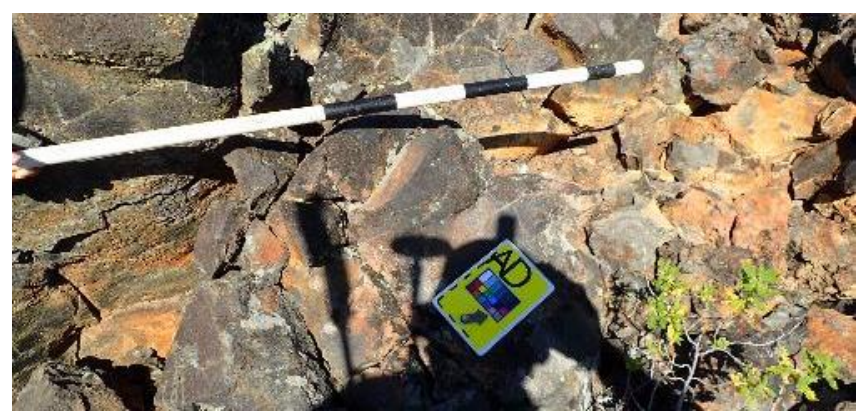

Figure 8. Temporary tags used to mark candidate sample locations; tags included an alphanumeric label, color bar, scale bar, and rotatable north arrow

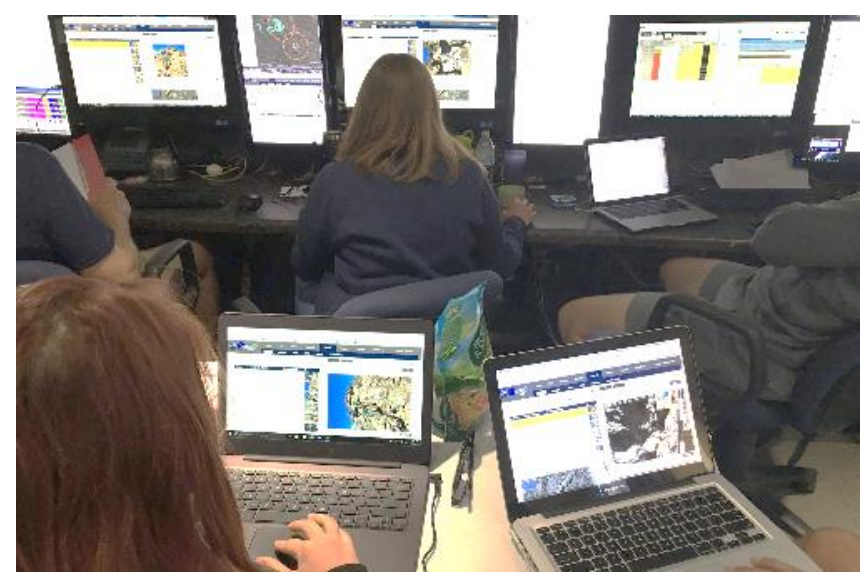

Figure 9. MSC members utilizing xGDS to record field notes and monitor video and still imagery during an EVA

In addition to the personnel roles detailed in Table 1, the MSC also included a Biology Lead, who provided feedback regarding features that may have an impact on habitability and/or the microbial community, a Geology Lead, who provided feedback on significant geological features, an Instrument Lead, who examined the spectral instrument data and offered additional information based on the instrument scans, an Imagery Lead, who carefully examined the details of the incoming still imagery, a Leaderboard Lead, who recorded the dynamic leaderboard priorities, alternatives, and rationale based on science team discussions, a Tactical Awareness Management Lead, who kept track of exactly where the EV crew were in the EVA timeline, and a Strategic Awareness Management Lead, who maintained general situational awareness in the context of the overall mission objectives and how the ongoing EVA activities may influence future EVAs.

\section{Out-of-Simulation EVA Support Personnel}

Each EVA was supported by a network of out-of-simulation support personnel. A Field Support Team (FST) assisted the EV crewmembers in the field and carried the science instruments and sampling tools. The FST Lead was responsible for leading the FST and coordinating all out-of-simulation activities, including those that occurred before, during, and after the EVA. The FST Instrument Aid provided the EV crew with technical instrument support. The FST Biology Sterilization Aid assisted the EV crew with maintaining sterilization during sampling tasks. The FST Steno/Runner recorded detailed field notes in the event of communication dropouts and assisted the FST Lead as needed. Two members of the BASALT Communications and Backpack Team provided communication infrastructure and backpack troubleshooting support in the field.

Inside the MSC, the simulation coordinator (SIMCOORD) communicated with the FST Lead to coordinate the start and end of each EVA. The MSC was further staffed with additional communications infrastructure and Minerva technical support personnel.

Table 3. Representative BASALT-1 planned EVA timeline

\begin{tabular}{|c|c|c|c|}
\hline 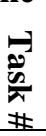 & $\begin{array}{l}\text { Planned } \\
\text { Duration } \\
\text { (hr:min) } \\
\end{array}$ & $\begin{array}{c}\text { Planned } \\
\text { Start Time } \\
\text { (hr:min) }\end{array}$ & Task \\
\hline 1 & $0: 45$ & $0: 00$ & Station A Approach \\
\hline 2 & 0:05 & $0: 45$ & $\begin{array}{l}\text { Station A Contextual } \\
\text { Survey }\end{array}$ \\
\hline 3 & $0: 30$ & $0: 50$ & $\begin{array}{l}\text { Station A Sample Loca- } \\
\text { tion Search }\end{array}$ \\
\hline 4 & $0: 15$ & $1: 20$ & Station B Approach \\
\hline 5 & 0:05 & $1: 35$ & $\begin{array}{l}\text { Station B Contextual } \\
\text { Survey }\end{array}$ \\
\hline 6 & $0: 30$ & $1: 40$ & $\begin{array}{l}\text { Station B Sample Loca- } \\
\text { tion Search }\end{array}$ \\
\hline 7 & $1: 00$ & $2: 10$ & Pre-Sampling Survey \\
\hline 8 & $0: 30$ & $3: 10$ & $\begin{array}{l}\text { Sampling at Sample Lo- } \\
\text { cation } 1\end{array}$ \\
\hline 9 & $0: 20$ & 3:40 & $\begin{array}{l}\text { Sampling at Sample Lo- } \\
\text { cation } 2\end{array}$ \\
\hline
\end{tabular}

Science Operations Research Data 
Objective and subjective data were collected during and after the EVAs to address the Science Operations research questions. Objective data included detailed EVA task timing information (such as planned versus actual task durations), which were collected and categorized in detailed task timing spreadsheets by out-of-simulation personnel so that researchers could investigate correlations between objective task performance and subjective ratings of acceptability and capability assessment. Other objective data included details surrounding the interactions between the crew and MSC (including the quality and type of interactions, the timing of the interactions relative to the EVA timeline, MSC assimilation time available prior to incurring crew idle time, and MSC assimilation time utilized), which were derived from dynamic leaderboard and Mission Log details, and network usage data, which parsed total bandwidth usage by data type.

Subjective data included a rigorous set of field-tested evaluation techniques were used to assess the ConOp and capabil-

\begin{tabular}{|c|c|c|c|c|}
\hline \multicolumn{5}{|c|}{$\begin{array}{l}\text { Acceptability Ratings should reflect the extent to which the co } \\
\text { to conducting human exploration and the extent to which impro }\end{array}$} \\
\hline \multicolumn{5}{|c|}{$\begin{array}{l}\text { Operational Acceptability: Able to reliably conduct operations } \\
\text { without excessive workload or (in-sim) avoidable inefficiencies }\end{array}$} \\
\hline \multicolumn{5}{|c|}{$\begin{array}{l}\text { Scientific Acceptability: Able to reliably complete and record s } \\
\text { with sufficient quantity, distribution, resolution, accuracy, and/ }\end{array}$} \\
\hline $\begin{array}{l}\text { Totally Accepta- } \\
\text { ble }\end{array}$ & \multicolumn{2}{|c|}{ Acceptable } & \multicolumn{2}{|c|}{ Borderline } \\
\hline $\begin{array}{l}\text { No improvements } \\
\text { necessary }\end{array}$ & \multicolumn{2}{|c|}{$\begin{array}{l}\text { Minor improve- } \\
\text { ments desired }\end{array}$} & \multicolumn{2}{|c|}{$\begin{array}{l}\text { Improvements war- } \\
\text { ranted }\end{array}$} \\
\hline 2 & 3 & 4 & 5 & 6 \\
\hline
\end{tabular}

ities employed during BASALT-1. This assessment methodology has been derived and refined through many previous NASA analog missions, including PLRP [12], RATS [7], and NEEMO [10], and provides a systematic, quantifiable approach to integrating and consolidating subjective results to inform functional and performance requirements for future exploration EVA design. BASALT-1 assessments included individual and consensus surveys of scientific, operational, and task acceptability to evaluate the overall ConOp, software systems, and communication protocols, and capability assessment ratings that described how essential or enabling a particular capability was envisioned to be for future Mars exploration EVAs. Simulation quality ratings were also collected to determine if the quality of the simulation itself was sufficient to allow for meaningful ratings of acceptability and capability assessment; simulation quality ratings of 4 or 5 meant that the simulation did not provide adequate conditions for meaningful evaluation. Acceptability and simulation quality definitions and rating scales are described in

2 Some simulation limitations or anomalies encountered, but mir

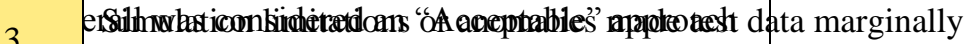

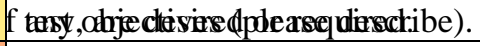

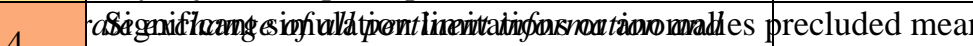
(please describe).

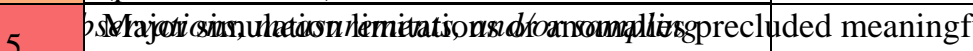

5 seribethe scientific hypothesis/hypotheses.

Unasieptable ble ing

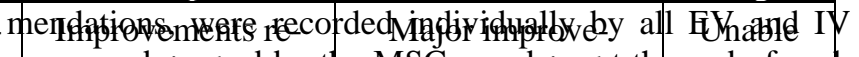

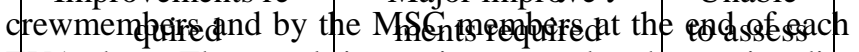
EVA phase. These real-time gating $\$$ servef as the starting discussion points for post-EVA consensus rating meetings,

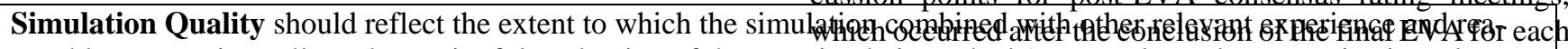

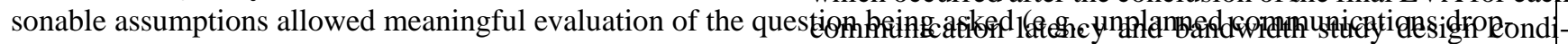
outs or unresolved hardware failures).

tion. During these consensus meetings, overall consensus ratRating Criteria

\begin{tabular}{l|l}
1 & $\begin{array}{l}\text { Simulation quality (e.g. hardware, software, procedury } \\
\text { or only minor ones that had no impact to the validity afotastidata. }\end{array}$
\end{tabular}

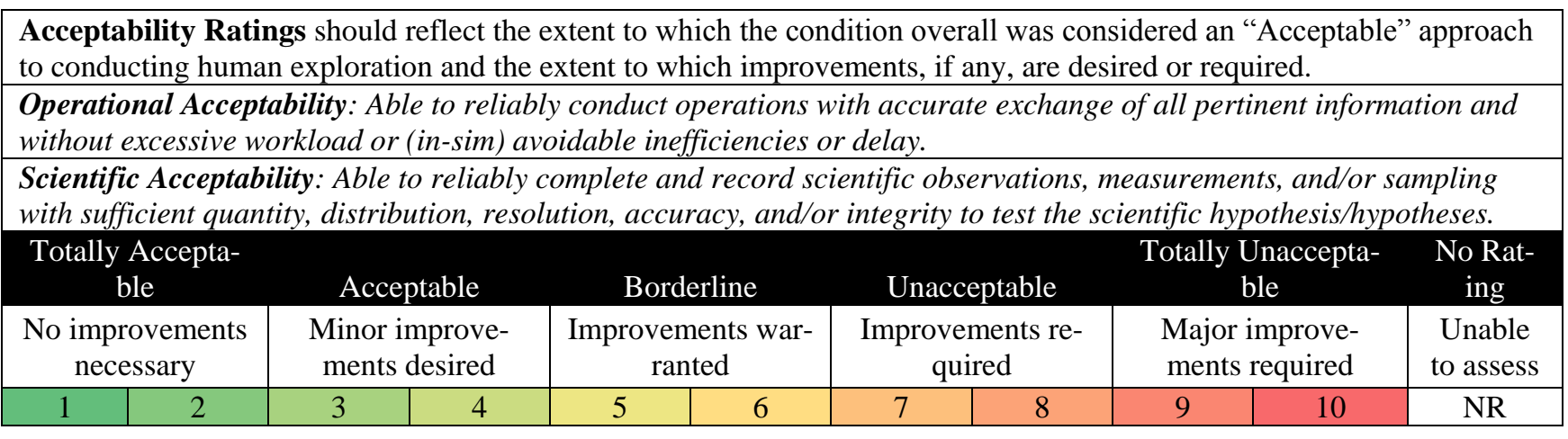

Simulation Quality should reflect the extent to which the simulation combined with other relevant experience and reasonable assumptions allowed meaningful evaluation of the question being asked (e.g., unplanned communications dropouts or unresolved hardware failures).

Rating Criteria

Simulation quality (e.g. hardware, software, procedures, comm., environment) presented either zero problems or only minor ones that had no impact to the validity of test data.

Some simulation limitations or anomalies encountered, but minimal impact to the validity of test data. 


\begin{tabular}{|c|l|}
\hline 3 & $\begin{array}{l}\text { Simulation limitations or anomalies made test data marginally adequate to provide meaningful evaluation of } \\
\text { test objectives (please describe). }\end{array}$ \\
\hline 4 & $\begin{array}{l}\text { Significant simulation limitations or anomalies precluded meaningful evaluation of major test objectives } \\
\text { (please describe). }\end{array}$ \\
\hline 5 & $\begin{array}{l}\text { Major simulation limitations or anomalies precluded meaningful evaluation of all test objectives (please de- } \\
\text { scribe). }\end{array}$ \\
\hline
\end{tabular}

Figure 10. BASALT-1 acceptability and simulation quality definitions and rating scales

\section{RESULTS AND DISCUSSION}

The BASALT-1 team deployed to the field several days in advance of the first planned EVA to set up and test the communications infrastructure, informatics backpacks, IV workstation, and MSC and to provide additional time for the EV crewmembers to practice the EVA science tasks in representative terrain that would be encountered during the actual EVAs. Limited-capability EVA dry runs were conducted under near-zero latency between the EV/IV crew and MSC during the two days leading up to the first planned EVA. After these dry run days, 10 consecutive days of EVAs (1 EVA per day) were executed. Error! Reference source not found. outlines the planned versus actual Science Operations communication study conditions laid out across the 10 EVAs. EVA Teams A and B alternated test days. EVAs 3 and 6-10 were completed under sufficient simulation quality to enable meaningful evaluations of the ConOp, software systems, and communication protocols. Simulation quality for EVAs 1, 2, 4 , and 5 were $\geq 4$ due to a combination of training effects, including inadequate crew and MSC personnel training, and poor communication quality (e.g., communication dropouts due to inadequate communication coverage in the field or problems routing capabilities to the MSC) during critical portions of the EVA. Therefore, since the length of the field deployment only allowed for 10 EVA days, only 3 study conditions were tested during BASALT-1: 5 min latency high bandwidth, 5 min latency low bandwidth, and 15 min latency high bandwidth.

Preliminary science plans were defined for each EVA prior to the start of the BASALT-1 deployment based on the precursor data used to plan EVA traverses. However, these plans were modified daily once in the field based on the cumulative science results from the EVAs to-date. Table 5 shows the updated science objectives for the BASALT-1 EVAs. As more EVAs were completed, the science objectives for each EVA became more specific to ensure that all science goals originally envisioned for BASALT-1 could be met.

Table 4. Planned versus actual latency and bandwidth conditions evaluated during BASALT-1; bold font indicates EVA completed under sufficient simulation quality to enable meaningful evaluation of the study condition

\begin{tabular}{c|cc|cc} 
EVA & \multicolumn{2}{|c|}{ planned } & \multicolumn{2}{c}{ actual } \\
(Team) & Lat. & BW & Lat. & BW \\
\hline $1(\mathrm{~A})$ & $5 \mathrm{~min}$ & high & $5 \mathrm{~min}$ & high \\
$2(\mathrm{~B})$ & $5 \mathrm{~min}$ & high & $5 \mathrm{~min}$ & high
\end{tabular}

\begin{tabular}{|c|c|c|}
\hline $3(\mathrm{~A})$ & $15 \mathrm{~min} \quad$ hig & $5 \mathrm{~min}$ \\
\hline $4(B)$ & $15 \mathrm{~min}$ & $5 \mathrm{~min}$ \\
\hline $5(\mathrm{~A})$ & $15 \mathrm{~min}$ & $\min$ \\
\hline $6(B)$ & $15 \mathrm{~min}$ & $\min$ \\
\hline 7 (A) & $5 \mathrm{~min}$ & $15 \mathrm{~min}$ \\
\hline $8(B)$ & $5 \mathrm{~min}$ & $15 \mathrm{~min}$ \\
\hline $9(\mathbf{A})$ & contingency & $5 \mathrm{~min}$ \\
\hline 10 (B) & contingency & $5 \mathrm{~min}$ \\
\hline
\end{tabular}

\section{EVA Timeline Results}

Figure 11 shows the planned versus actual EVA timelines for the EVAs executed under adequate simulation quality. In general, these EVAs were completed approximately on-timeline, with the exception being EVA 3, which required a $1 \mathrm{hr}$ extension for sampling due to the difficulty encountered by the EV crew when attempting to extract the desired unaltered basalt. Most stations had sufficient scientific features of interest to warrant spending at least the planned time exploring during the contextual survey and sample location search, and so EV crew were often faced with balancing detailed scientific investigations with operational time constraints. Occasionally, however, the EV crew would arrive at a station that did not meet any of the BASALT-1 scientific objectives; this was typically a consequence of the low-resolution precursor data being insufficient for detailed planning. In these instances, the crew either spent more time at the other station planned for that EVA or explored a new neighboring region.

\section{Table 5. Science objectives for each BASALT-1 EVA}

\begin{tabular}{cl} 
EVA & Science Objectives \\
\hline 1 & 1. Highest local alteration \\
& 2. Lowest local alteration \\
& 3. Moderate local alteration \\
\hline 2 & 1. Rubbly pahoehoe with highest hot alteration \\
& 2. Smooth pahoehoe with highest hot alteration \\
& 3. Unaltered rubbly pahoehoe \\
& 4. Unaltered smooth pahoehoe \\
\hline 3 & 1. Highest alteration within levee \\
& 2. Lowest alteration within levee \\
& 3. Highest alteration within channel \\
\hline 4 & 1. Highest local alteration \\
& 2. Low density frothy lava of any alteration \\
& level \\
& 3. Mid-grade local alteration OR frothy lava
\end{tabular}




\begin{tabular}{ll}
\hline 5 & 1. Highest local alteration \\
& 2. Lowest local alteration \\
& 3. TOP: frothy-textured lava \\
\hline 6 & 1. Highest local alteration for full sample suite \\
& 2. Lowest local alteration for geology and ar- \\
chive samples only & 3. Moderately altered geology sample \\
\hline 7 & 1. Highest local alteration \\
\hline 8 & 1. Unaltered full sample suite \\
& 2. Moderately altered geology and archive sam- \\
ples
\end{tabular}

During each EVA, the MSC was faced with two critical nolater-than "deadlines" in which MSC input regarding presampling and sampling recommendations had to be sent to the EV/IV crew so that they would not incur any idle time waiting on ground input. These deadlines were based on the current communication latency. Hence, assuming EV1 and EV2 were operating on timeline, the MSC needed to send pre-sampling and sampling guidelines no later than 5 or 15 min prior to the start of these phases. However, with the dynamic leaderboard approach, the MSC is encouraged to send multiple pre-sampling and sampling priority rankings. In theory, these rankings can be sent every time the MSC modifies the leaderboard (although in practice, the MSC often takes a more moderate approach that considers what additional information they are expecting to receive from EV in the nearterm and how tasked they perceive the IV crewmembers might be). The advantage of the dynamic leaderboard and sending important updates to the crew is that if the crew happen to start working ahead in the timeline or if the communication network encounters dropouts, the crew can still have some understanding of the MSC priorities and rationales. In Figure 11, the black triangles and diamonds on the planned timelines show the no-later-than deadlines for MSC pre-sampling and sampling input, respectively. The white triangles and diamonds on the actual timelines show when dynamic leaderboard pre-sampling and sampling updates, respectively, were sent from the MSC to the crew. For each of these EVAs, at least one pre-sampling and one sampling dynamic leaderboard was sent to the crew, and hence no crew idle time was ever incurred waiting on pre-sampling or sampling recommendations from the MSC. However, the MSC strategy regarding when to send dynamic leaderboard updates changed over the course of the field deployment: initially, more updates were sent earlier, whereas later, fewer updates were sent. This change was likely due to the communication network became more stable later in the mission, and so the MSC was less concerned with potential communication dropouts, and the observation by the MSC that the EV crew consistently worked either on-timeline or slightly behind-timeline, but not ahead of timeline.

Figure 12 depicts how EV spent their time during each phase of the EVA (including time spent translating, conducting surveys [verbal descriptions and capturing still imagery and video footage], using scientific instruments, preparing for sampling [donning gloves and performing biology sterilization procedures], sampling, troubleshooting, and other [outof-sim time allocated to collecting real-time acceptability ratings]) and how these times varied across EVAs and conditions. Together with Figure 11, these results demonstrate how EVA plans required flexibility to account for both lessons learned during this first field deployment and the variety of science objectives needing to be met. As the BASALT-1 team learned how long it took to effectively complete the tasks within each EVA phase, planned EVA timelines were updated for subsequent EVAs. For instance, the sample location search was lengthened from $15 \mathrm{~min}$ to $30 \mathrm{~min}$ to facilitate additional search time to better meet science objectives; providing the EV crew with a longer sample location search allowed them to explore the station in greater detail and recommend a larger number candidate samples, which gave the MSC a better understanding of overall characteristics of the station itself and more candidate sample options to choose from. Furthermore, pre-sampling tasks were modified later in the deployment. The pre-sampling phases of EVAs 3 and 6 incorporated the use of two scientific instruments; later EVAs only incorporated one of these instruments, as the other was found to be not as helpful as originally anticipated for identifying the best candidate samples. However, the time originally allocated to using that second instrument was replaced by having the EV crew break into near-by basalt to examine, image, and describe the interiors of candidate samples. Additionally, science locations of interest and science priorities changed from day to day. Some approaches required more time, as the distance to and between stations were greater. Some samples were expected to be more difficult to extract than others, and so longer sampling phases were planned.

Table 6 summarizes the number of candidate samples tagged, pre-sampled, and sampled for each EVA. Full sample suites included 7 total samples, such that both geologic and biologic science objectives were met; in some instances, full suites were not needed and only partial suites were collected. In general, the number and type of samples collected during BASALT-1 were sufficient to meet the BASALT science objectives for this deployment; further insight regarding the quality of the samples collected and how this maps to the BASALT Science objectives is currently under investigation. No significant differences were noted in pre-sampling or sampling productivity as a function of latency or bandwidth. 
Table 6. Number of candidate sample locations tagged, pre-sampled, and sampled for each BASALT-1 EVA

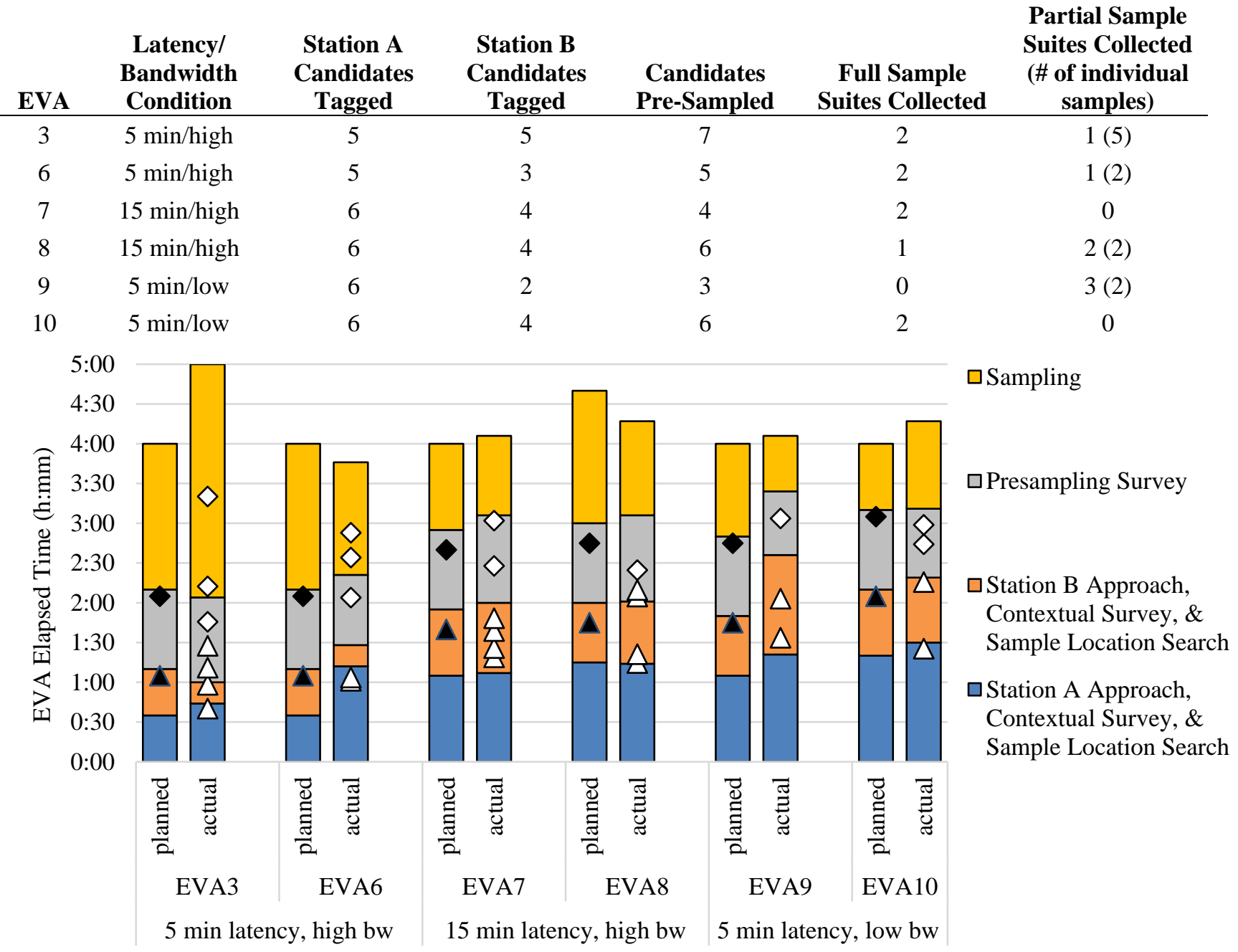

Figure 11. Planned and actual BASALT-1 EVA timelines; black triangles and diamonds represent the no-later-than deadline for the MSC to send pre-sampling and sampling guidance, respectively; white triangles and diamonds represent actual MSC pre-sampling and sampling recommendations, respectively 

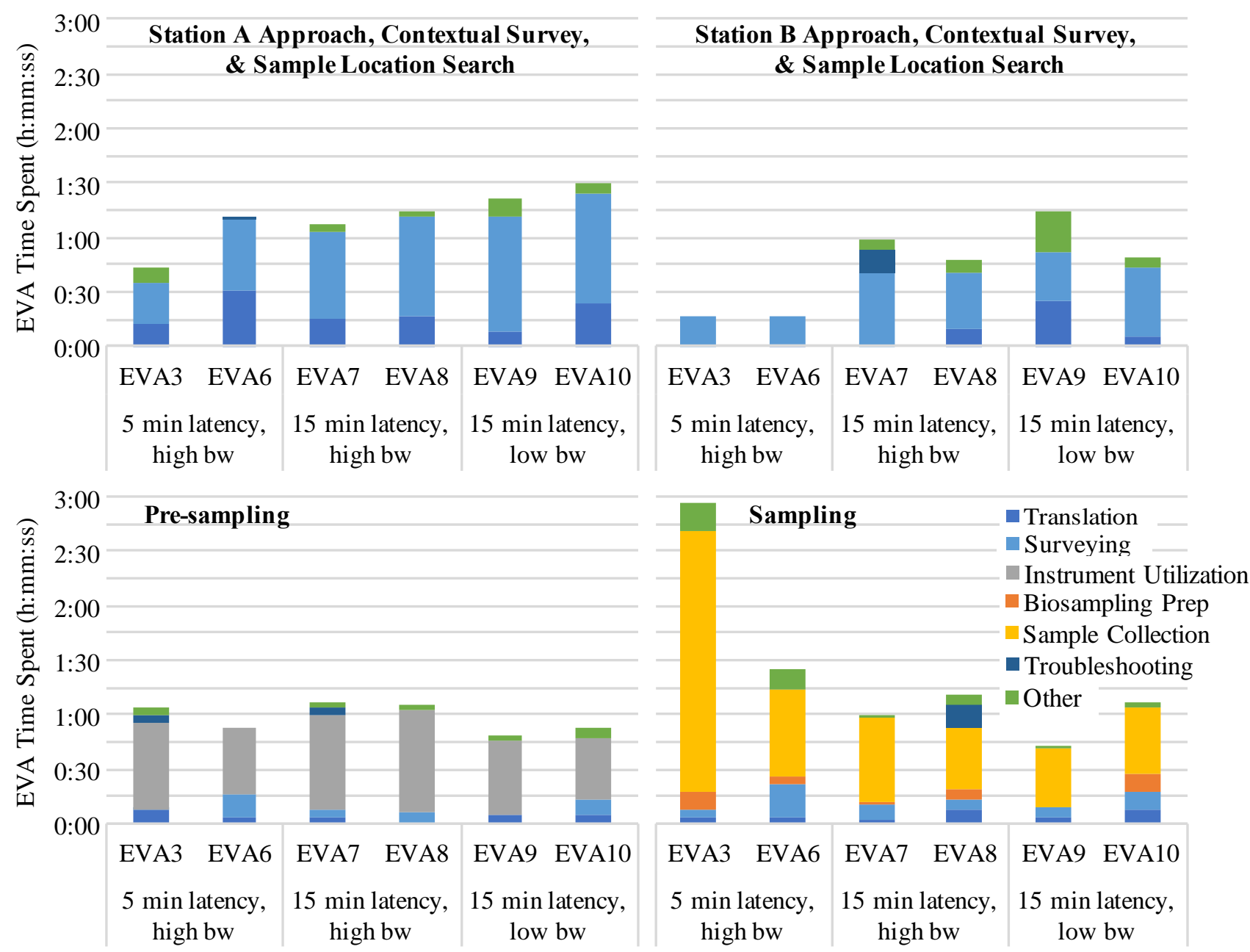

Figure 12. Categorization of time spent by phase of EVA during BASALT-1

\section{Consensus Acceptability Ratings}

The overall scientific and operational acceptability ratings and the scientific and operational acceptability ratings broken down by EVA phase are presented in Figure 13 and Figure 14 , respectively. These data were collected separately by the EV/IV crews and by the MSCs during consensus meetings with the two EVA teams after the second EVA for the given communication study condition was completed. During these meetings the EV/IV crews and MSCs were asked to consider whether the BASALT-1 baseline ConOp, software systems, and communication protocols worked acceptably during real scientific field exploration, whether the level of acceptability changed with communication latency or bandwidth condition, and to describe corresponding improvements that were desired, warranted, or required (Science Ops research questions $1 \mathrm{~A}$ and $1 \mathrm{~B}$ ). While the primary goal was to obtain a single scientific and single operational acceptability rating (with associated comments, including specific improvements) that holistically considered the baseline ConOp, software systems, and communication protocols for each communication study condition, thinking about so many components simultaneously proved to be highly challenging by all. So, for the BASALT-1 deployment, the ratings were first broken down by EVA phase, and the EV/IV crews and MSCs were asked to consider the acceptability of each phase individually. After this was accomplished, the EV/IV crews and MSCs then used the by-phase ratings to decide upon an overall rating.

The station approach phase was deemed acceptable (minor improvements desired) by the EV/IV crews and MSC scientists, regardless of communication study condition. The MSC scientists noted that clearer, more systematic verbal descriptions and additional still imagery would have been helpful for them to better understand the surrounding terrain and be better suited to assist the crew in looking for potential TOPs, but the MSC scientists did not believe that having these additional details would have substantially altered their decisions during the later EVA phases. Notably, however, the MSC operators rated the station approach phase as borderline (improvements warranted) for the high bandwidth study conditions and unacceptable (improvements required) for the low bandwidth study condition. Because this phase was associated with a substantial amount of traversing, and sometimes through treacherous terrain, the MSC operators perceived this phase to pose a potentially larger operational risk than the other phases. Because of the limited verbal descriptions and still imagery coming from the field, the MSC operators had a 
hard time understanding where the crew were, especially in relation to potentially hazardous features. The MSC operators noted that having the mobile SA camera follow the crew during the approach phases (instead of only being used once the crew arrived on station) would have been an important capability to augment the footage coming from the much narrower field-of-view EV video cameras. The MSC operators also noted that this phase would have been more challenging to execute under low bandwidth conditions without video, and so a significant increase in still imagery of the surrounding terrain to offset the loss of video would have been required.

The station contextual survey phase was rated borderline (improvements warranted) for both scientific and operational acceptability by the EV/IV crews, regardless of communication latency or bandwidth condition. The contextual survey was intended to provide a quick, high-level overview of the station in relation to the science objectives expected to be met at that station. However, due to the facts that only 5 min were allocated for this task and that the crew conducted the entire survey from the perimeter of the station at whatever location the station approach phases ended (sometimes this was up higher on a ridge overlooking the station, while other times this was near the bottom of a ravine where it was difficult to view the station in its entirety), the quality of contextual surveys the crew felt they could provide were highly terrain dependent. Hence, warranted scientific and operational improvements included accounting for station-specific terrain details when planning the amount of time necessary to more acceptably complete this phase, including time allocations, for example, for the crew to traverse the entire perimeter to provide better contextual descriptions and imagery. The MSCs generally rated the contextual survey phase as borderline (improvements warranted) scientifically, noting that the mobile SA and EV chest cameras were insufficient for capturing all scientifically relevant features of the station in the manner in which the EV crew conducted the contextual surveys; ratings were slightly less acceptable for the low bandwidth condition where additional still imagery was recommended to account for the lack of video footage. The MSCs also recommended placing the mobile SA camera on a higher stand to provide better top-down views of the station as a whole and using color correction algorithms to enhance the still and video footage, which would have been especially useful under challenging lighting conditions where direct sunlight and shadows were sometimes problematic. The MSCs rated the contextual survey phase as operationally acceptable (minor improvements desired) for the high bandwidth conditions and operationally borderline (improvements warranted) for the low bandwidth condition; the primary operational improvement recommended was to either adjust the timeline to allow for a longer contextual survey (as this task consistently ran long) or adjust expectations for what could realistically be accomplished during a 5 min task.

The sample location search phase was rated scientifically and operationally acceptable (minor improvements desired) by the EV/IV crews for all latency and bandwidth conditions. From the crew perspective, their task during this phase was to systematically search the station for as many candidates as they could find that met that the science objectives for that particular EVA. In general, they did not operate differently for one communication condition versus another. Minor improvements desired were establishing better strategies to divide and conquer among EV1 and EV2 to maximize search capabilities within the allocated time and being more vigilant with still imagery to minimize glare and shadows; however, both of these improvements could have been attributed to limited crew training, and hence could have been more accurately captured under reduced simulation quality, as opposed to reduced acceptability. From the MSCs' perspective, on the other hand, the sample location search was generally rated scientifically and operationally unacceptable (improvements required). The MSC scientists noted that because the EV crew were operating at a rapid pace laying down candidate sample markers and pushing a substantial amount of still imagery and video footage, it was difficult to keep up with the action in the field while simultaneously having the necessary side discussions to generate their pre-sampling survey leaderboard. While some improvements could have been attributed to training effects, such as needing better organizational structure within the MSC to more effectively distribute task loading, the scientists also noted that a higher mobile SA camera that provided a better top-down view of the station as a whole would have enabled the scientists to better understand where the candidate markers were located relative to one another; they surmised that this capability alone would have helped them rank leaderboard priorities more quickly. Importantly, the MSC scientists noted that the EVA timeline, which had to operate across latency and bandwidth constraints, did not allow for sufficient ground assimilation time to accomplish all of the science objectives originally planned for each of EVA. Hence, there was a change in science strategy between the first $6 \mathrm{EVAs}$ (corresponding to the $5 \mathrm{~min}$ latency high bandwidth condition) and last 4 EVAs (corresponding to the 15 min latency high bandwidth condition and 5 min latency low bandwidth condition) in that the earlier EVAs included 3 separate science objectives, whereas the later EVAs only focused on 1 general science objective (see Table 5). This strategy change is an important BASALT-1 lesson learned, although it did partially confound the acceptability data, as can be seen in the improvement in scientific acceptability from the 5 min latency high bandwidth condition (EVAs 3 and 6) to the 15 min latency high bandwidth condition (EVAs 7 and 8). However, the scientists stated that they would not have been able to reliably keep up with the crew had they not made this strategy change. On the operational side, the MSCs struggled with how and when to best convey information that was rapidly changing on their end to the IV crewmembers. They requested better tools be developed and implemented to facilitate this transfer of information.

The pre-sampling survey phase was rated scientifically and operationally acceptable (minor improvements desired) by the EV/IV crews for all latency and bandwidth conditions. From their perspective, the improvement desired was a better navigational tool that enabled them to more easily return to 
specific candidate sample markers; this was partially resolved after EVA 6 when the IV crewmembers began dropping candidate sample location marker pins on the traverse map that the EV crew could then view on their wrist display. However, the pre-sampling survey was rated scientifically unacceptable (improvements required) by the MSC scientists. For this phase, it was critical for the scientists to receive the raw spectral instrument data (as opposed to only still images of the instrument results screens) in order for this data to be most useful for informing sampling priorities. The MSC operators also rated this phase unacceptable (improvements required) because substantial inefficiencies were induced when requiring the EV crew to dictate detailed mineralogy reports and take additional images of the instrument screens. The capability to stream the raw instrument data back to the MSC was an improvement required for future BASALT deployments.

The sampling phase was rated totally acceptable (no improvements necessary) scientifically and operationally by the EV/IV crews for all latency and bandwidth conditions. The MSCs, however, rated this phase borderline (improvements warranted) or unacceptable (improvements required) scientifically and operationally for all latency and bandwidth conditions. On the scientific side, it was noted that the EV crew needed to be provided with better sampling tools, such as battery powered drills and sledges, to more easily and efficiently extract the desired samples of the correct size. Additionally, the scientists wanted better situational awareness regarding where on the outcrop exactly the rock was being extracted; better imaging protocols needed to be developed and implemented. Furthermore, the timeline design itself made it difficult for the MSCs to effectively influence the sampling phase; this was especially true under the longer latency, since by the time the MSCs received information from the field regarding sampling, it was generally too late to do anything about. Operationally, it was difficult for the MSCs to gauge how long a given sampling task would take. In general, unaltered basalt was more challenging to extract and typically took longer, but occasionally altered basalt was just as difficult. The 7 samples that made up a full sample suite had different mass requirements, and so harvesting samples of the correct size added complexity. In general, the EV/IV crews were less concerned with the sampling phase running long since they were always under conditions in which they could extend the EVA. However, the MSCs had a very limited understanding of how the crew was doing, including with respect to fatigue near the end of a long EVA, and so they had difficulty recommending (or not recommending) EVA extensions; better tools to assist with this were noted.

Overall scientific and operational acceptability ratings were derived by the EV/IV crews and the MSCs from the EVAby-phase acceptability data. The EV/IV crews consistently rated the baseline ConOp, software systems, and communication protocols as acceptable with minor improvements desired; this was true for both scientific and operational acceptability for each of the 3 study conditions. Operationally, the
MSCs rated the high bandwidth conditions as borderline (improvements required), with the 5 min latency condition being slightly more acceptable than the 15 min latency condition, and the low bandwidth condition as unacceptable (improvements required). The MSC operators noted that they had better situational awareness under high bandwidth conditions and were more able to influence the EVA when the latency was smaller. However, because of the warranted or required improvements detailed for the individual EVA phases, the overall operational acceptability was borderline or unacceptable. Scientifically, the MSC rated the 5 min latency high bandwidth condition as acceptable (improvements required) and the 5 min latency low bandwidth and 15 min latency high bandwidth conditions as borderline (improvements warranted). The longer EVA phases (including the sample location search, pre-sampling, and sampling) were rated as scientifically unacceptable for the $5 \mathrm{~min}$ latency high bandwidth condition, which is what drove the overall scientific acceptability score for this study condition to be unacceptable. Importantly, however, the difference in scientific acceptability ratings between the 5 min latency high bandwidth EVAs and the EVAs associated with the later-tested latency and bandwidth conditions was more likely due to training effects than differences in this particular latency and bandwidth combination, as noted during post-deployment debriefs. EVAs 3 and 6 were the first EVAs in which the MSC scientists worked together, and a substantial amount of learning occurred during those early EVA days: MSC scientists, who were used to being in the field themselves, found themselves in a new advisory role only and were limited by the performance of the EV crew and capabilities afforded to them. Individual roles and responsibilities were adjusted within the MSC and EVA science objectives were scaled back to enable the scientists to adapt to this new way of operating. While some of these required improvements should have been captured in the simulation quality scores, it was difficult for the scientists to separate these lessons learned from the acceptability ratings, since they were being collected mid-deployment while the learning process was ongoing.

\section{Study Limitations and Primary Lessons Learned}

The BASALT-1 field deployment was the first of three currently planned for the BASALT project, and there were several study limitations, as well as many important lessons learned. Due to limited availability of BASALT personnel and travel budgets prior to the BASALT-1 deployment, all hardware and software capabilities could not be tested in an integrated, operational environment in advance. As a result, there were significant communications and networking issues that occurred between the EV crew in the field, the IV crew in the IV workstation, and the MSC during the early EVAs. These issues precluded meaningful evaluation of the research questions for 4 of the $10 \mathrm{EVAs}$, which also resulted in one study condition (15 min latency low bandwidth) not being able to be assessed during BASALT-1, as originally planned. During the remaining 6 EVAs, some communication dropouts occurred, which occasionally made it difficult to assess all intended capabilities thoroughly. Furthermore, because all 
capabilities were not fully operational during the training and engineering dry run days prior to the first EVA day, various training effects were observed throughout the deployment: personnel became more familiar with their required roles and responsibilities from the first EVA to the last EVA, and best practices and strategies evolved from the start of the deployment to the end. Future BASALT deployments will prioritize integrated hardware and software testing in the field prior to the first EVA and minimal changes will be made to the personnel role assignments until all study conditions have been thoroughly evaluated to take advantage of the training achieved during BASALT-1.

Another important lesson learned revolves around the man- ner in which the scientific and operational acceptability consensus ratings were considered and collected. The intent of the BASALT project Science Operations research questions is to identify ConOps and capabilities enable scientific return under the operational constraints required for human exploration missions so that results from the BASALT project can help inform the design of future Mars EVAs. Hence, to adequately address this intent, the acceptability ratings should be considered under the thought process of a larger Mars-forward umbrella, as opposed to a BASALT-specific one. The challenge with doing this during BASALT-1 was that critical personnel training was ongoing during the first half of the field deployment, and capabilities were consistently being refined in the field.

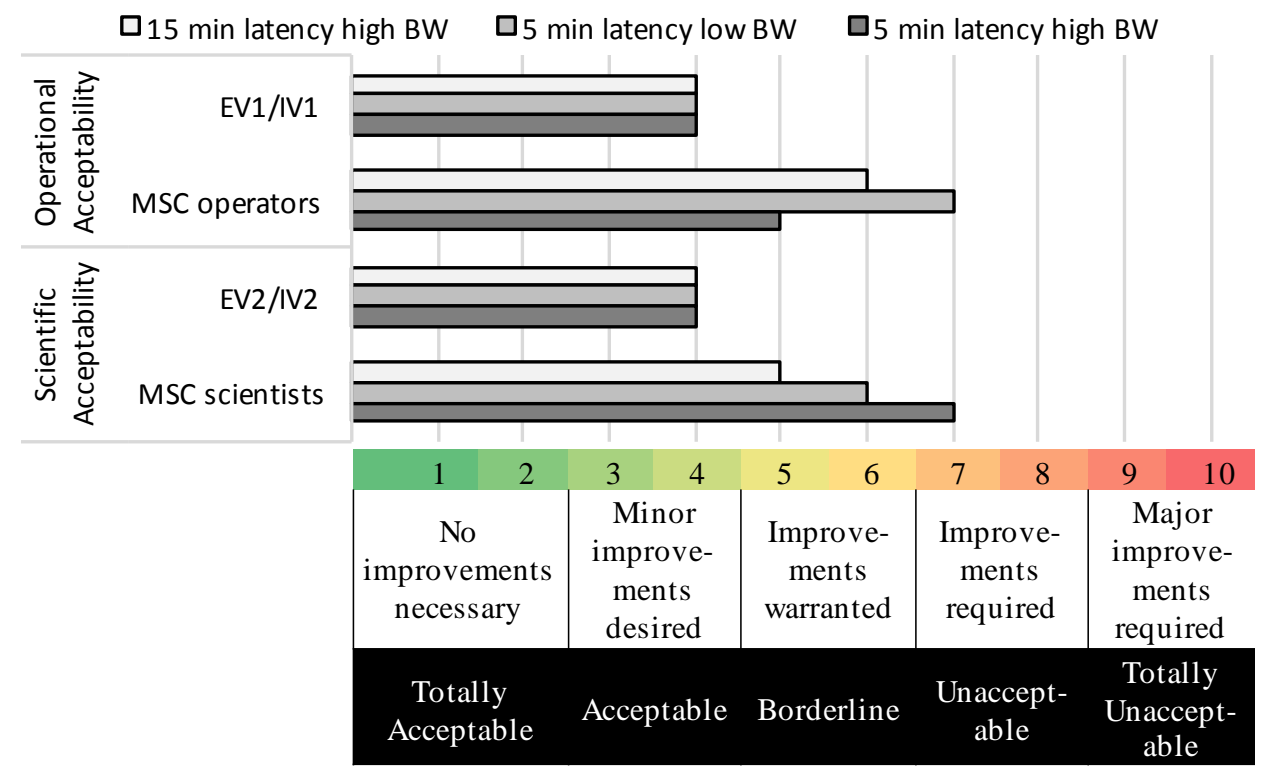

Figure 13. BASALT-1 overall scientific and operational acceptability as a function of latency and bandwidth 


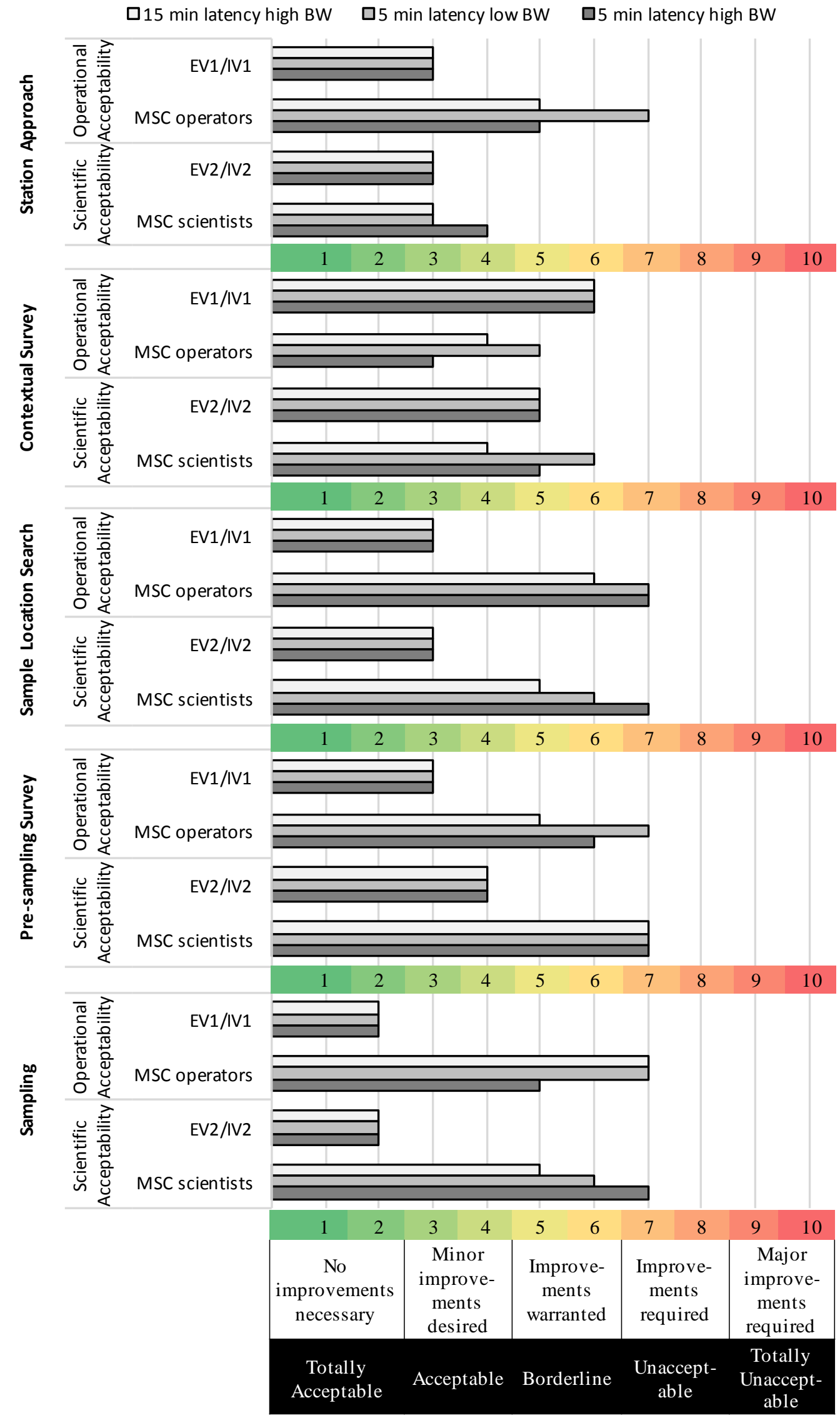

Figure 14. BASALT-1 scientific and operational acceptability by EVA phase as a function of latency and bandwidth 
Hence, the focus of many post-EVA consensus meetings were BASALT-specific discussions that needed to be closed out before the larger Mars-forward discussions could occur. In some ways, the BASALT-1 field deployment was an extensive engineering and operational test evaluation, where personnel were thoroughly trained, hardware and software bugs were worked out, and initial discussions regarding Mars-forward capabilities were practiced. BASALT-1 was highly successful in this regard, and so future field deployments will focus on more advanced discussions that more closely meet the Science Operations goals of the BASALT project.

\section{Conclusions}

Vetted design principles and operational methodologies for managing communication latencies and bandwidth limitations are critical for mitigating risks associated with future Mars human exploration missions. BASALT-1 was the first of several field deployments for the BASALT project, in which the primary Science Operations goal was to critically evaluate various concepts of operations and capabilities in light of future human exploration missions to Mars. Preliminary results indicate scientific and operational improvements are both warranted and required for the tested ConOp and capabilities. A significant number of lessons were learned, which will inform future BASALT field deployments and ultimately future space systems that will enable effective and efficient human scientific exploration of Mars.

\section{REFERENCES}

[1] D. A. Craig, P. Troutman, and N. Herrmann, "Pioneering Space Through the Evolvable Mars Campaign," in AIAA SPACE 2015 Conference and Exposition, 2015, p. 4409.

[2] M. C. Deans, D. Lees, T. Smith, T. Cohen, T. Morse, and T. Fong, "Field testing next-generation ground data systems for future missions," in Proc. Lunar and Planetary Sci. Conf.(LPSC), Houston, TX, 2011.

[3] J. J. Marquez et al., "Supporting Real-Time Operations and Execution through Timeline and Scheduling Aids," in 43rd International Conference on Environmental Systems, American Institute of Aeronautics and Astronautics, 2013.

[4] A. L. Gilkey et al., "Evaluation of a Surface Exploration Traverse Analysis and Navigation Tool," in 41st International Conference on Environmental Systems, 2011, p. 5181.

[5] A. F. Abercromby, M. L. Gernhardt, and J. Jadwick, "Evaluation of dual multi-mission space exploration vehicle operations during simulated planetary surface exploration," Acta Astronautica, vol. 90, no. 2, pp. 203-214, 2013.

[6] A. F. Abercromby, S. P. Chappell, and M. L. Gernhardt, "Desert RATS 2011: Human and robotic exploration of near-Earth asteroids," Acta Astronautica, vol. 91, pp. 34-48, 2013.

[7] A. F. J. Abercromby, S. P. Chappell, H. L. Litaker, M. L. Reagan, and M. Gernhardt, "NASA Research and Technology Studies (RATS) 2012: Evaluation of human and robotic systems for exploration of near-Earth asteroids," presented at the 43rd International Conference on Environmental Systems, Vail, CO, July 2013, 2013.

[8] S. P. Chappell, A. F. Abercromby, and M. L. Gernhardt, "NEEMO 15: Evaluation of human exploration systems for near-Earth asteroids," Acta Astronautica, vol. 89, pp. 166-178, 2013.

[9] S. P. Chappell, A. F. J. Abercromby, M. Reagan, and M. Gernhardt, "NEEMO 16: Evaluation of systems for human exploration of near-Earth asteroids," presented at the AIAA 43rd International Conference on Environmental Systems, Vail, CO, July 2013, 2013.

[10] S. P. Chappell et al., "NEEMO 18-20: Analog Testing for Mitigation of Communication Latency During Human Space Exploration," presented at the IEEE Aerospace Confernce, Big Sky, MT, 2016.

[11] D. S. S. Lim et al., "A historical overview of the Pavilion Lake Research Project-Analog science and exploration in an underwater environment," Geological Society of America Special Papers, vol. 483, pp. 85-115, 2011.

[12] M. J. Miller et al., "PLRP-3: Operational Perspectives of Conducting Science-Driven Extravehicular Activity with Communications Latency," 2016.

[13] A. F. Abercromby, S. Chappell, D. Lee, A. Howe, and M. Gernhardt, "Human Exploration of Phobos," in Proceedings of the IEEE Aerospace Conference, Big Sky, MT, 2015.

M. L. Gernhardt et al., "Human exploration missions to phobos prior to crewed mars surface missions," in 2016 IEEE Aerospace Conference, 2016, pp. 1-20: IEEE.

[15] D. W. Beaty et al., "An analysis of the precursor measurements of Mars needed to reduce the risk of the first human missions to Mars," Unpublished white paper, vol. 77, 2005. 
[16] R. Yingst, B. Cohen, D. Ming, and D. Eppler, "Comparing Apollo and Mars Exploration Rover (MER)/phoenix operations paradigms for human exploration during NASA Desert-RATS science operations," Acta Astronautica, vol. 90, no. 2, pp. 311-317, 2013.

[17] E. Jones. (2004, February 16). The Apollo Lunar Surface Journal. Available: http://www.hq.nasa.gov/office/pao/History/alsj/

[18] P. Clark, "Revolution in field science: Apollo approach to inaccessible surface exploration," Earth, Moon, and Planets, vol. 106, no. 2-4, pp. 133-157, 2010.

[19] M. M. Connors, D. B. Eppler, and D. G. Morrow, "Interviews with the Apollo Lunar Surface Astronauts in Support of Planning for EVA Systems Design, TM-108846," National Aeronautics and Space Administrations, Houston, TX1994.

[20] G. G. Schaber, "The US Geological Survey, branch of Astrogeology-a chronology of activities from conception through the end of Project Apollo (19601973)," 2331-1258, 2005.

[21] W. B. Garry and J. E. Bleacher, Analogs for planetary exploration. Geological Society of America, 2011.

[22] S. G. Love and M. L. Reagan, "Delayed voice communication," Acta Astronautica, vol. 91, no. 0, pp. 89-95, 10// 2013.

\section{BIOGRAPHY}

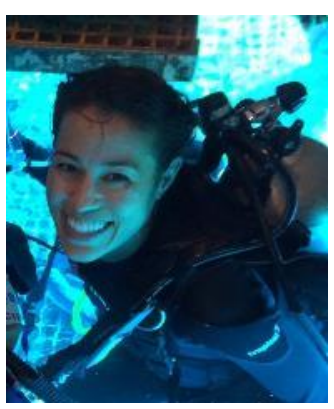

Kara Beaton received her bachelor's and master's degrees in aerospace engineering from the University of Illinois and Massachusetts Institute of Technology, respectively. She completed her doctoral studies in biomedical engineering at the Johns Hopkins University School of Medicine. She has extensive experience with operationally driven aerospace and biomedical research with the US Navy, NASA, and various clinical laboratories. She is currently a research engineer in the EVA Physiology Laboratory at JSC, where she is actively involved in the Exploration Analogs and Mission Development team. Kara is currently serving as the science operations co-lead for the BASALT project.

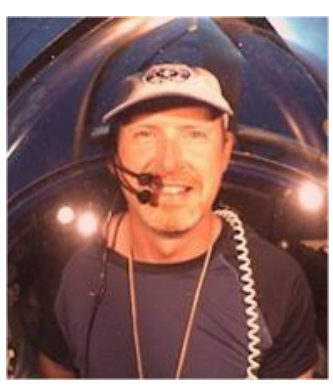

Steve Chappell attended the University of Michigan and earned a bachelor's degree in aerospace engineering. He also earned master's and doctoral degrees from the University of Colorado in aerospace engineering sciences, researching human performance and spacesuit systems in simulated reduced gravity. His career has spanned many areas of engineering and science, including work on embedded software for fighter aircraft, satellite ground systems development, and Earth-observing satellites systems engineering. In recent years, in addition to helping lead the Mars Moons Human Spaceflight Architecture Team, his work focused on optimizing human and system performance for the next generation of space exploration. He has extensive experience leading and taking part in research in multiple exploration analog environments including arctic, desert, underwater, alpine, and partial gravity simulators. He currently is the science operations research co-lead for BASALT.

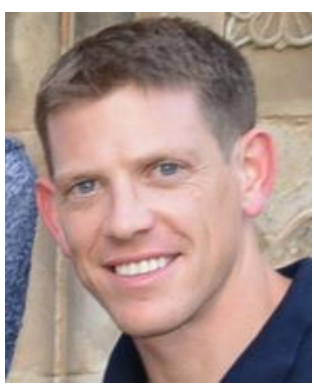

Andrew Abercromby received an M.Eng. in mechanical engineering from the University of Edinburgh during which he worked on X-38 in the Flight Mechanics Laboratory at Johnson Space Center (JSC). He earned a Ph.D. in motor control from the University of Houston while working in the JSC Neurosciences Laboratory. Andrew is now the lead of NASA's EVA Physiology Laboratory and also serves as EVA Scientist for the Biomedical Research and Environmental Sciences Division. His current research focuses on measurement and optimization of human performance and operations in extreme exploration environments and includes research studies in desert, ocean, lake, virtual reality, 
Arctic, and Antarctic environments, including experience in saturation and under-ice scientific research diving. Andrew is the deputy PI for the BASALT project.

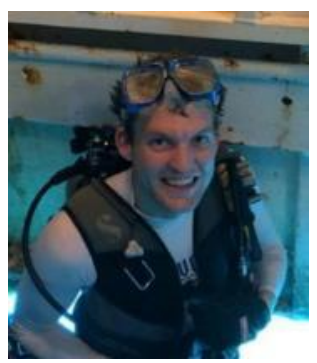

Matthew James Miller is a graduate research assistant in the Cognitive Engineering Center at the Georgia Institute of Technology. He has interned at the NASA Johnson Space Center, Charles S. Draper Laboratory, The Aerospace Corporation, and ATA Engineering. He received a B.S. and a M.S. in Aerospace Engineering from Georgia Institute of Technology in 2012 and 2014, respectively, and is currently pursuing his Ph.D. His research interests are in the areas of decision making and decision support development in complex work domains. He is supported by a NASA Space Technology Research Fellowship and is advised by Dr. Karen Feigh. Matthew is part of the BASALT science operations team.

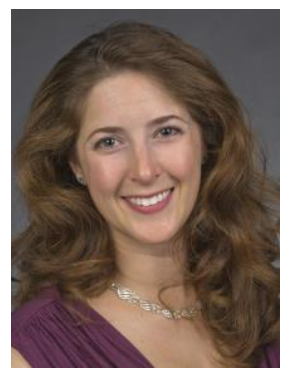

Shannon Kobs Nawotniak is a physical volcanologist with a focus on spatial patterns associated with eruption products. She uses field work, lab experiments, and computational modeling to link those spatial patterns to processes associated with eruptions, from lava flow behavior to atmospheric mixing in eruption columns. She completed her BS in Geology at Michigan Technological University and her PhD in Geology at the University at Buffalo. Shannon is the deputy PI and geology co-lead for the BASALT project.

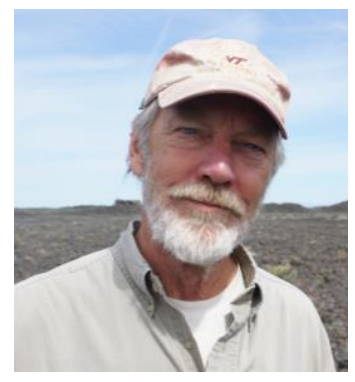

Scott Hughes received a B.S. from Virginia Tech, an M.S. from Northern Arizona University, and a Ph.D. in from Oregon State University $(O S U)$, specializing in volcanology, petrology, geochemistry, and planetary geology. As a NASA post-doc at OSU, and later as geosciences faculty member at Idaho State University (ISU), his research focused on the geochemical analyses and trace element modeling of lunar basalts, mare volcanic glasses, and terrestrial basalts. His primary efforts are geared toward the evaluation of basalt lava flows, geochemical processes during magma genesis and evolution, and the growth of small shield volcanoes and their surrounding lava fields on the eastern Snake River Plain. Now Professor Emeritus at ISU, his analog research on terrestrial lava flows continues in order to evaluate models that help bridge the connections between magma genesis and emplacement processes on the Moon and Mars. Scott is the geology co-lead for the BASALT project.

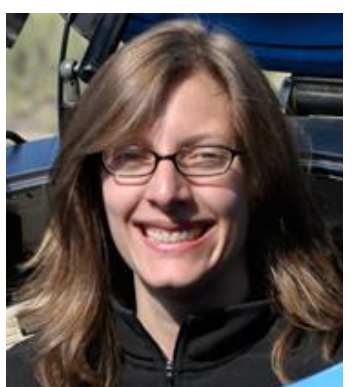

Allyson Brady is an organic geochemist interested in using organic and isotopic biomarkers to characterize microbial communities living in extreme environments. With over 10 years of field experience, she has conducted field work in remote, extreme environments including geothermal hot springs, alkaline lakes, a perennially ice-covered lake in the Antarctic, and has piloted submersibles in an underwater analogue environment. She is currently a post-doctoral research fellow in the School of Geography and Earth Sciences at McMaster University. Allyson is the organic geochemistry lead for the BASALT project.

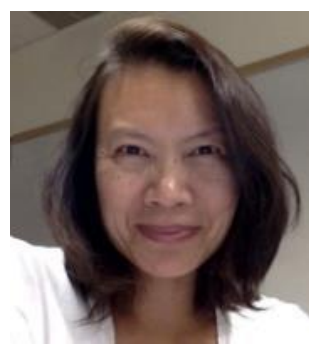

Darlene Lim is a geobiologist who has extended her research focus to include the development of operations concepts and capabilities in support of human-robotic scientific exploration. She has since spent over two decades leading field research around the world, in locations as remote as the Arctic and Antarctic, as well as in various underwater environments where she has spent hours piloting submersibles as a scientist and explorer. Darlene earned her Ph.D. in geobiology at the University of Toronto and is currently serving as the principal investigator of the BASALT project.

\section{Acknowledgements}

The authors wish to thank and acknowledge the many scientists and engineers who contributed to the analysis described in this report. The authors also wish to thank Jennifer Tuxhorn and Jackie Reeves for their thorough reviews. 